\title{
The growth rate of trajectories of a quadratic differential
}

\author{
HOWARD MASUR† \\ Department of Mathematics, University of Illinois at Chicago, Chicago, \\ Illinois 60680, USA
}

(Received 18 February 1987, revised 14 December 1987)

\begin{abstract}
Suppose $q$ is a holomorphic quadratic differential on a compact Riemann surface of genus $g \geq 2$. Then $q$ defines a metric, flat except at the zeroes. A saddle connection is a geodesic joining two zeroes with no zeroes in its interior. This paper shows the asymptotic growth rate of the number of saddles of length at most $T$ is at most quadratic in $T$. An application is given to billiards.
\end{abstract}

\section{Introduction}

Let $X$ be a closed Riemann surface of genus $g>1$ and $q=q(z) d z^{2}$ a nonzero holomorphic quadratic differential on $X$ such that $\int_{X}|q|=1$. A trajectory is an arc along which $\arg q(z) d z^{2}$ is constant. The trajectory is regular if it does not contain zeroes of $q$ and singular if the two endpoints of the arc are zeroes and there are no zeroes in the interior of the arc. Singular trajectories are also called saddle connections. If there is a closed regular trajectory there is a parallel family of freely homotopic trajectories of equal $\left|q^{1 / 2} d z\right|$ length sweeping out an annulus. On the boundary of the annulus are saddle connections. A geodesic for the metric $\left|q^{1 / 2} d z\right|$ is made up of pieces of trajectories that make an angle of at least $\pi$ at a zero. Between any two points there is a unique geodesic in every homotopy class.

This paper is concerned with the asymptotics of the number of parallel families of closed regular trajectories and the number of saddle connections. Specifically, let $N_{1}(T)$ denote the number of families of length $\leq T$ and $N_{2}(T)$ the number of saddle connections of length $\leq T$. Since the boundary of the annulus contains a saddle connection of smaller length, clearly $N_{1}(T) \leq N_{2}(T)$.

THEOREM 1.

$$
\varlimsup_{T \rightarrow \infty} \frac{N_{1}(T)}{T^{2}} \leq \varlimsup_{T \rightarrow \infty} \frac{N_{2}(T)}{T^{2}}<\infty
$$

Remark 1. This result is perhaps surprising in that the growth rate does not depend on the genus. For example, for any metric the growth rate of homotopy classes of simple curves of length $\leq T$ is $T^{68-6}$ ([R] Lemma 2.4). However the geodesic for $\left|q^{1 / 2}\right||d z|$ in a homotopy class is often made of many pieces of saddle connections 
so the number of saddle connections of length $\leq T$ is less than the number of geodesics, in fact grows at most quadratically. The question of lower bounds will be investigated in a later paper.

Remark 2. For $g=1$ there are of course no singular trajectories. Suppose $X$ is $\mathbf{C}$ modulo $z \rightarrow z+1$ and $z \rightarrow z+i$. Then a trajectory of $q=d z^{2}$ is closed if the tangent of the argument is a rational, $p / q$, and the length is $\left(p^{2}+q^{2}\right)^{1 / 2}$. Then $N_{1}(T)$ is the set of integer lattice points $(p, q)$ inside a disc of radius $T ; p, q$ relatively prime. It is easy to see that $N_{1}(T)$ grows quadratically with $T$.

We give an application to the study of billiards. Let $\Delta$ be a connected polygon in the plane. A broken line formed by line segments $\left[x_{0}, x_{1}\right],\left[x_{1}, x_{2}\right], \ldots,\left[x_{n-1}, x_{n}\right]$ will be called a generalized diagonal of $\Delta$ if it lies inside $\Delta$ except for the points $x_{i}$. The points $x_{0}, x_{n}$ are vertices, $x_{1}, \ldots, x_{n-1}$ lie on the sides and for $i=1, \ldots, n-1$ the segments $\left[x_{i-1}, x_{i}\right]$ and $\left[x_{i}, x_{i+1}\right]$ form the same angle with the side of $\Delta$ on which $x_{i}$ lies. The number of generalized diagonals is always infinite.

Katok [K] raised the question of the asymptotic count of generalized diagonals. Let $D_{T}(\Delta)$ be the number of length $\leq T$. He proved

$$
\varlimsup_{T \rightarrow \infty} \frac{\log D_{T}(\Delta)}{T}=0 .
$$

Let $G$ be the group generated by reflection in lines through the origin parallel to the sides. If $G$ is finite $\Delta$ is said to be a rational billiard table.

THEOREM 2. $\quad \varlimsup_{T \rightarrow \infty} \frac{D_{T}(\Delta)}{T^{2}}<\infty$ if $\Delta$ is rational.

It was shown by Boshernitzan [B] that Theorem 2 implies that the geodesic flow on a rational billiard table is uniquely ergodic in almost every direction, a result first proved in [K-M-S].

It is easy to see that classical integrable billiards such as a rectangle, equilateral triangle satisfy the quadratic estimate $D_{T}(\Delta) \sim c T^{2}$. This was generalized by Gutkin [G] to a broader class of 'almost integrable' billiards.

Finally let $P_{T}(\Delta)$ be the number of parallel families of periodic orbits of length $\leq T$ for a rational billiard. Since $P_{T}(\Delta) \leq D_{T}(\Delta)$ we have

Corollary.

$$
\varlimsup_{T \rightarrow \infty} \frac{P_{T}(\Delta)}{T^{2}}<\infty .
$$

Theorem 2 is a direct consequence of Theorem 1 once it is understood how a billiard flow on a rational polygon gives rise to trajectories of a quadratic differential on a closed Riemann surface. A generalized diagonal gives rise to a saddle connection. This is described in detail in [K-M-S].

The idea behind Theorem 1 is as follows. We will fix certain constants $\varepsilon>0$, $0<\sigma<1$ and $C>1$ and consider saddle connections $\beta$ whose length is in the interval $[\sigma n, n]$. It is enough to show there are $0\left(n^{2}\right) \operatorname{such} \beta$. For each $\beta$ we will choose the argument so that $\arg q(z) d z^{2}$ is $\pi$ along $\beta$; that is, $\beta$ is a vertical trajectory. We then contract the length of $\beta$ to $\varepsilon$ via a Teichmüller map which contracts along vertical trajectories and expands along horizontal trajectories. On this image 
Riemann surface under the Teichmüller map, if every saddle connection crossing $\beta$ has length $\geq C \varepsilon$ we will say $\beta$ is $\varepsilon$-isolated and $\varepsilon$-wide on the domain surface. A simple calculation, Proposition 2.1, shows there is a lower bound of at least constant divided by $n^{2}$ between the arguments of crossing $\varepsilon$-wide saddle connections and thus $O\left(n^{2}\right)$ such saddle connections, where the bound depends only on constants.

For example, suppose we have a parallel family of closed trajectories of length $T$ on a torus. When the length is contracted to $\varepsilon$, any closed curve crossing has length $\geq 1 / \varepsilon$ since the family fills out the surface and the area is one. For suitably chosen $C, \varepsilon$ this is $\geq C \varepsilon$.

On a surface of higher genus the analogous statement may be false since the area of a parallel family may be much less than one; it may fill up only a small part of the surface. For any such $\beta_{0}$ we will associate a sequence $Y_{1}, \ldots, Y_{p}$ of surfaces where $p$ is bounded in terms of the genus. Each $Y_{i}$ will have a boundary of saddle connections and a distinguished saddle connection $\beta_{i}$ on it. Each $\beta_{i}$ will be in the interior of $Y_{i+1}$ for $i=0, \ldots, p-1$.

After a suitable Teichmüller map the saddle connections on the boundary of $Y_{i}$ can simultaneously be made short while $\beta_{p}$ itself is $\varepsilon$-wide. This allows us to use Proposition 2.1 to count the number of $\beta_{p}$ that occur and since $\beta_{p}$ is an element of a system of curves that simultaneously become short, we will be able (Proposition 2.5 ) to count the number of boundaries of $Y_{p}$, hence the number of $Y_{p}$ that occur.

Next we will compute (Theorem 4.1) for a given surface with boundary the number of saddle connections in the interior of a given length. This will allow us for each $Y_{p}$ to compute the number of $\beta_{p-1}$ that occur and again by Propoisition 2.5 the number of $Y_{p-1}$ for given $Y_{p}$. The product of this with the number of $Y_{p}$ gives the total number of $Y_{p-1}$. We then proceed inductively to find the number of $\beta_{0}$ that define any sequence. The technical difficulties of this paper stem from defining such sequences and more specifically considering not just a single saddle connection but systems of saddle connections. Accordingly $\S 1$ contains preliminary definitions and constructions necessary for the construction of the $Y_{i} . \S 2$ contains the computations for $\varepsilon$-wide saddle connections, $\S 3$ the above mentioned construction of the $Y_{i}$ and $\$ 4$ the computations that are the proofs of the main theorems.

I am grateful to referees for numerous helpful suggestions.

\section{Preliminaries}

This section is devoted to definitions and various constructions needed later in the proof of Theorem 4.1 and the main theorem. For the reader unfamiliar with quadratic differentials we refer to $[\mathbf{S}]$ as a basic reference and to $[\mathbf{K}-\mathbf{M}-\mathbf{S}]$ as well.

In the remainder of the paper, $Y$ with subscripts will refer to a (variable) compact Riemann surface of genus $p$ and $n \geq 0$ boundary components satisfying $2 p-2+n \leq 2 g-2$ and $\varphi=\varphi d z^{2}$ will refer to a quadratic differential on $Y$ with $\|\varphi\|=\int_{Y}|\varphi|=1$. We require the boundary $\partial Y$ to be a union of saddle connections. $Y$ will often contain a distinguished saddle connection $\alpha$, possibly in its interior. 
For any saddle connection $\beta$ of $\varphi$ define $h(\beta)=\int_{\beta}\left|\operatorname{Re} \varphi^{1 / 2} d z\right|$ and $v(\beta)=\int\left|\operatorname{Im} \varphi^{1 / 2} d z\right|$. They are referred to as the horizontal and vertical lengths, respectively. The length $\int_{\beta}|\varphi|^{1 / 2}|d z|=|\beta|=\left(h^{2}(\beta)+v^{2}(\beta)\right)^{1 / 2}$.

Two saddle connections are disjoint if they intersect at most at one or two common zeroes. Otherwise they cross. A basic fact used throughout this paper is that there are at most $C_{1}=C_{1}(p, n)$ disjoint singular trajectories independent of the metric. A system $\Gamma=\left(\gamma_{1}, \ldots, \gamma_{p}\right)$ will always refer to a collection of disjoint trajectories and $|\Gamma|=\max \left|\gamma_{i}\right|, v(\Gamma)=\max v\left(\gamma_{i}\right)$.

The basic tool in this paper is the Teichmüller map. For $t \in \mathbf{R}$ let $f_{t}: Y \rightarrow Y$, denote the Teichmüller map determined by $\varphi$ with maximal dilatation $K=e^{t}$. Denote by $\varphi_{i}$ the unit norm terminal quadratic differential on $Y_{t}$. We denote by $|\beta|_{t}, v_{s}(\beta)$, $h_{t}(\beta)$ the corresponding length functions on $Y_{t}$. They are given by:

$$
h_{l}(\beta)=\mathrm{e}^{t / 2} h(\beta), v_{t}(\beta)=\mathrm{e}^{-t / 2} v(\beta),|\beta|_{\imath}=\left(h_{t}^{2}(\beta)+v_{t}^{2}(\beta)\right)^{1 / 2} .
$$

Let $\varphi_{\theta}=\mathrm{e}^{\mathrm{i} \theta} \varphi$. Then $h_{\theta, t}(\beta), v_{\theta, t}(\beta)$ and $|\beta|_{\theta, t}$ will denote the effect on $\beta$ of first multiplying by $\mathrm{e}^{\mathrm{i} \theta}$ and then applying a Teichmüller map. If $\theta$ is understood we will drop that subscript. The absence of a $t$ variable refers to $t=0$. Denote by $\theta_{\beta}$ the angle for which $h_{\theta_{\beta}}(\beta)=0$. We call $\theta_{\beta}$ the vertical angle for $\beta$. Lengths of course are not invariant under $\varphi \rightarrow \varphi$, but areas are.

We next define the cross product of two saddle connections. Define

$$
\left|\beta_{1} \times \beta_{2}\right|=\left|\int_{\beta_{1}} \operatorname{Re} \varphi^{1 / 2} d z \int_{\beta_{2}} \operatorname{Im} \varphi^{1 / 2} d z-\int_{\beta_{2}} \operatorname{Re} \varphi^{1 / 2} d z \int_{\beta_{1}} \operatorname{Im} \varphi^{1 / 2} d z\right| .
$$

Note that the absolute value does not depend on choice of branch of $\varphi^{1 / 2}$ along $\beta_{i}$ nor does it depend on the orientation of $\beta_{i}$. Notice also $\left|\beta_{1} \times \beta_{2}\right|$ is invariant under both $\varphi \rightarrow \varphi_{\theta}$ and $\varphi \rightarrow \varphi_{1}$.

A notion basic for counting saddle connections on a surface with boundary is the following.

Definition. Let $D>0$. We say $\beta_{1}$ and $\beta_{2}$ are $D$-close if $\left|\beta_{1} \times \beta_{2}\right| \leq D$. If $\Gamma$ is a system of curves, $\beta$ is $D$-close to $\Gamma$ if $\beta$ is $D$-close to each $\gamma \in \Gamma$.

LemMA 1.1. Suppose $\alpha$ is $D_{1}$-close to $\beta$ and $\beta$ is $D_{2}$-close to $\gamma$. Further suppose $|\alpha| \leq M_{1}|\beta|$ and $|\gamma| \leq M_{2}|\beta|$. Then $\alpha$ is $M_{1} D_{2}+M_{2} D_{1}$-close to $\gamma$.

Proof. Since cross products and lengths, ||, are invariant under rotations $\varphi \rightarrow \varphi_{\theta}$, assume $h(\beta)=0, v(\beta)=|\beta|$. Then $h(\alpha) \leq D_{1} /|\beta|, \quad h(\gamma) \leq D_{2} /|\beta|$. This implies $|\alpha \times \gamma| \leq v(\alpha) h(\gamma)+h(\alpha) v(\gamma) \leq M_{1} D_{2}+M_{2} D_{1}$.

The next lemma says that if $\beta$ is close to $\alpha$, and $\gamma$ and $\beta$ can simultaneously be made short then $\gamma$ is close to $\alpha$.

LEMMA 1.2. Suppose $\beta$ is D-close to $\alpha$ and $|\beta| \geq|\alpha| / M_{1}$. Further suppose for some $t>0, \quad M_{2}$ and $\gamma, \varepsilon / 2 \leq v_{t}(\beta) \leq|\beta|_{t} \leq \varepsilon$ and $|\gamma|_{1} \leq M_{2} \varepsilon$. Then there is $a$ $D^{\prime}=D^{\prime}\left(M_{1}, \varepsilon, M_{2}, D\right)$ such that $\gamma$ and $\alpha$ are $D^{\prime}$-close.

Proof. Since $t>0$ and $|\beta|_{t} \leq \varepsilon$ we have $h(\beta) \leq \varepsilon$. Then

$$
v^{2}(\beta) \geq|\beta|^{2}-\varepsilon^{2} \geq|\alpha|^{2} / M_{1}^{2}-\varepsilon^{2} \geq \frac{v^{2}(\alpha)}{M_{1}^{2}}-\varepsilon^{2} \text {. }
$$


Thus $v(\alpha) \leq M_{1}(v(\beta)+\varepsilon)$ so $v_{t}(\alpha) \leq M_{1}\left(v_{t}(\beta)+\varepsilon\right) \leq 2 M_{1} \varepsilon$. Then $h_{t}(\beta) v_{t}(\alpha) \leq$ $2 M_{1} \varepsilon^{2}$. Since closeness is preserved under $\varphi \rightarrow \varphi_{1}$ and $\beta$ and $\alpha$ are $D$-close, $v_{t}(\beta) h_{t}(\alpha) \leq D+v_{t}(\alpha) h_{t}(\beta) \leq D+2 M_{1} \varepsilon^{2}$. Thus

$$
h_{t}(\alpha) \leq \frac{2 D+4 M_{1} \varepsilon^{2}}{\varepsilon} \text {. }
$$

We conclude that

$$
|\gamma \times \alpha| \leq v_{t}(\gamma) h_{i}(\alpha)+v_{t}(\alpha) h_{t}(\gamma) \leq\left(M_{2} \varepsilon\right)\left(\frac{2 D+4 M_{1} \varepsilon^{2}}{\varepsilon}\right)+\left(M_{2} \varepsilon\right)\left(2 M_{1} \varepsilon\right) .
$$

Suppose $\Gamma_{1}$ and $\Gamma_{2}$ are two systems with mutually disjoint saddle connections.

Definition. A system $\Gamma$ separates $\Gamma_{1}$ from $\Gamma_{2}$ if every arc intersecting both $\Gamma_{1}$ and $\Gamma_{2}$ intersects $\Gamma$. $\Gamma$ is allowed to contain saddle connections of $\Gamma_{i}$ but no saddle connection of $\Gamma$ crosses a saddle connection of $\Gamma_{i}$. A system $\Gamma$ properly separates if $\Gamma \neq \Gamma_{i}$ and if $\Gamma_{i}$ is a single saddle connection $\beta, \beta \notin \Gamma$.

Definition. $\Gamma M$ - separates $\Gamma_{1}$ from $\Gamma_{2}$ if it separates and $|\Gamma|<\left|\Gamma_{2}\right| / M$.

We will use this notion often when $\Gamma_{1}$ and $\Gamma_{2}$ are saddle connections $\beta$ and $\alpha . \beta$ is not $M$-separated from $\alpha$ if there is no $\Gamma$ separating $\beta$ from $\alpha$ with $|\Gamma|<|\alpha| / M$.

We will often need to combine two systems to form a new one.

Proposition 1.3. Suppose $\Gamma_{1}$ and $\Gamma_{2}$ are each systems dividing $Y$ into two or more components. There is a system $\Gamma$ called the combination of $\Gamma_{1}$ and $\Gamma_{2}$ with the properties that

(i) if $\alpha$ is $D_{i}$-close to $\Gamma_{i}$ then $\alpha$ is $C_{1}\left(D_{1}+D_{2}\right)$-close to $\Gamma$.

(ii) $|\Gamma| \leq C_{1}\left(\left|\Gamma_{1}\right|+\left|\Gamma_{2}\right|\right)$,

(iii) if $\alpha$ is disjoint from $\Gamma_{1}$ and $\Gamma_{2}$ it is disjoint from $\Gamma$ and $\Gamma$ separates $\alpha$ from each $\Gamma_{i}$.

Proof. Denote by $*$ juxtaposition of two arcs. Consider a component $U$ of the complement of $\Gamma_{1} \cup \Gamma_{2}$ and $\Omega$ a component of the boundary of $U$. $\Omega$ consists of a union of subarcs of saddle connections of $\Gamma_{i}$. First we include in $\Gamma$ any arc of $\Omega$ that is an entire saddle connection. Now suppose $\gamma_{1} \in \Omega$ is a proper subarc of a saddle connection of $\Gamma_{1}$, with one endpoint a nonzero endpoint of a proper subarc $\gamma_{2} \in \Omega$ of a saddle connection in $\Gamma_{2}$. Replace $\gamma_{1} * \gamma_{2}$ by the geodesic $\gamma$ in $U$ in the same homotopy class joining the endpoints of $\gamma_{1} * \gamma_{2}$. Now consider $\gamma$ to be in $\Omega$ instead of $\gamma_{1} * \gamma_{2}$ thereby reducing $U . \gamma$ is a union of saddle connections and subarcs of trajectories.

Notice if $\alpha$, a saddle connection, is disjoint from $\gamma_{1}$ and $\gamma_{2}$ it is disjoint from $\gamma$. Moreover any arc from $\alpha$ to $\gamma_{1}$ in $U$ must cross $\gamma$. If $\gamma$ is a simple closed regular trajectory, replace $\gamma$ in $U$ by the geodesics of saddle connections in the homotopy class on the boundary of the annulus. Again if $\alpha$ is disjoint from $\gamma_{1}$ and $\gamma_{2}$ it is disjoint from $\gamma$ and any arc in $U$ to $\gamma_{1}$ crosses $\gamma$. If $\gamma$ is not a closed trajectory, include all saddle connections of $\gamma$ in $\Gamma$. If there is an arc $\sigma$ of $\gamma$ which is not a saddle connection, an endpoint of $\sigma$ is a nonzero endpoint of an $\operatorname{arc} \gamma_{1}^{\prime} \in \Omega$ of $\Gamma_{1}$ or $\gamma_{2}^{\prime} \in \Omega$ of $\Gamma_{2}$. Replace $\sigma * \gamma_{1}^{\prime}$ (respectively $\sigma * \gamma_{2}^{\prime}$ ) by the geodesic $\gamma^{\prime}$ in the 
homotopy class joining the endpoints. We continue in this manner of replacing a juxtaposition of arcs with geodesics $\gamma^{\prime}$ until one of three possibilities occurs.

(i) $\gamma^{\prime}$ is a saddle connection which we then include in $\Gamma$.

(ii) $\gamma^{\prime}$ is a closed regular trajectory. Include in $\Gamma$ the geodesic of saddle connections homotopic to $\gamma^{\prime}$, in $U$.

(iii) $\Omega$ reduces to a triangle and none of the arcs are saddle connections. In that case eliminate all such arcs.

This process is repeated for each subarc for each $\gamma_{i}$ of $\Omega$ not previously eliminated and then for each boundary component $\Omega$ of each complementary $U$ to form $\Gamma$. We have seen that if $\alpha$ is disjoint from $\Gamma_{i}$ it is disjoint from $\Gamma$. We next show $\Gamma$ separates $\alpha$ from $\Gamma_{1}$. Suppose $\sigma$ is an arc from $\alpha$ to $\Gamma_{1}$ missing $\Gamma$ and hitting $\alpha$ and $\Gamma_{1}$ only at its endpoints. If $\alpha$ is in $U$ and $\sigma$ stays in $U$ except for its endpoints then we have seen $\sigma$ must intersect $\Gamma$. Thus $\sigma$ must leave $U$ a first time crossing a subarc $\gamma_{2}$ of $\Gamma_{2}$. It must therefore intersect a curve $\gamma$ homotopic to $\gamma_{1} * \gamma_{2}, \gamma_{1} \in \Gamma_{1}$. If $\gamma$ itself is not in $\Gamma$ then $\sigma$ must cross a curve homotopic to $\gamma * \gamma_{1}^{\prime}$ or $\gamma * \gamma_{2}^{\prime}$ where $\gamma_{i}^{\prime} \in \Gamma_{i}$ since by assumption $\sigma$ does not cross $\gamma_{i}^{\prime}$ as it leaves $U$ for the first time at $\gamma_{2}$. Continuing, this argument shows $\sigma$ intersects a $\gamma \in \Gamma$ proving (iii).

Now if $\gamma$ is constructed out of pieces $\gamma_{1} \in \Gamma_{1}$ and $\gamma_{2} \in \Gamma_{2}$ then $|\alpha \times \gamma| \leq$ $\left|\alpha \times \gamma_{1}\right|+\left|\alpha \times \gamma_{2}\right|$. Now for each saddle $\beta \in \Gamma_{i}$ written as

$$
\beta=\gamma_{i}^{1} * \gamma_{i}^{2} * * \gamma_{i}^{p},
$$

a juxtaposition of pieces of $\beta,|\alpha \times \beta|=\sum_{j=1}^{p}\left|\alpha \times \gamma_{i}^{j}\right|$. Since there are at most $C_{1}$ saddle connections in $\Gamma_{i}$, for any $\gamma \in \Gamma,|\alpha \times \gamma| \leq C_{1}\left(D_{1}+D_{2}\right)$ proving (i).

Further each $\gamma$ constructed out of $\gamma_{1}$ and $\gamma_{2}$ satisfied $|\gamma| \leq\left|\gamma_{1}\right|+\left|\gamma_{2}\right|$. Since $\Gamma_{1}$ and $\Gamma_{2}$ consist of at most $C_{1}$ saddle connections each, we have each $\gamma \in \Gamma$ satisfies $|\gamma| \leq C_{1}\left(\left|\Gamma_{1}\right|+\left|\Gamma_{2}\right|\right)$, proving (ii).

Remark. If all components of the complement of $F_{1} \cup F_{2}$ are simply connected and contain no saddle connections, then $\Gamma=\varnothing$. This possibility will not occur in this paper.

Corollary 1.4. Suppose $\Gamma_{i}, i=1, \ldots, p, p \leq C_{1}$ are dividing systems $\left|\Gamma_{i}\right| \leq M$ and $\alpha$ is disjoint from each. Then there is a system $\Gamma$, which combines the $\Gamma_{i} ; \Gamma$ separates $\alpha$ from each $\Gamma_{i} ; \Gamma$ and $\alpha$ are disjoint and $|\Gamma| \leq\left(2 C_{1}\right)^{C_{1}} M$.

Proof. Take the combination of $\Gamma_{1}$ and $\Gamma_{2}$, the combination of that with $\Gamma_{3}$ and so forth.

Our next objective is Proposition 1.6, a construction which will be used in Theorem 4.1 to reduce a general counting problem to counting only saddle connections that are $D$-close to each other, $D$ a universal number depending only on the genus not on the quadratic differential. We begin with

Lemma 1.5. Suppose $\beta_{1}$ and $\beta_{2}$ bound two sides of a triangle with no vertices in the interior. Then $\left|\beta_{1} \times \beta_{2}\right| \leq 2$.

Proof. $\left|\beta_{1} \times \beta_{2}\right|$ is the area of a parallelogram which is twice that of the triangle. The triangle has at most area one. 
Now an arbitrary pair of saddle connections may not form two sides of a triangle and therefore may not be 2-close. In fact there is no universal $D$ such that any two saddle connections are $D$-close. The aim here is to prove.

Proposition 1.6. Assume $\beta$ and $\alpha$ are disjoint saddle connections on $Y$ and $\beta$ is not $M$-separated from $\alpha$. Then there are $D, M_{1}$ depending on $C_{1}, M$ and a sequence

$$
\beta=\beta_{0}, \beta_{1}, \ldots, \beta_{n}=\alpha \quad n \leq C_{1}
$$

of disjoint saddle connections such that $\beta_{i}$ is D-close to $\beta_{i+1}$ and is not $M_{1}$-separated from it $i=0, \ldots, n-1$.

We first prove

Proposition 1.7. Suppose $\beta$ is a saddle connection, $\Omega_{0}$ is a system such that either $\beta \in \Omega_{0}$ or they are disjoint. Further assume for some $M,|\beta| \geq\left|\Omega_{0}\right| / M$. Then for any $M^{\prime}>1$ there is a $D=D\left(M, M^{\prime}, C_{1}\right)$ such that if $\beta$ is not $D$-close to $\Omega_{0}$ there is a $\Omega_{0}^{\prime}$ properly separating $\beta$ from $\Omega_{0}-\beta$ such that $|\beta| \geq M^{\prime}\left|\Omega_{0}^{\prime}\right|$ and $\beta D$-close to $\Omega_{0}^{\prime}$.

Proof. If $\beta$ is $D=2$-close to $\Omega_{0}$ we have nothing to show. Suppose not. The proof will consist of adding and eliminating disjoint saddle connections at most $C_{1}$ times. The constant $D$ produced may increase by a fixed multiplicative constant each time. The final constant will not depend on $|\beta|$ or $\left|\Omega_{0}\right|$, only on $M, M^{\prime}$ and $C_{1}$. We will refer to all such constants by $D$ even as then change.

We begin by considering triangles with $\beta$ as one edge whose other edges are either disjoint from $\Omega_{0}-\beta$ or coincide with saddle connections in $\Omega_{0}-\beta$. Any such edge is 2 -close to $\beta$ by Lemma 1.5 .

If we can separate $\beta$ from $\Omega_{0}-\beta$ by $\Gamma_{1}$ by adding 1 or 2 such triangles whose longest side $\gamma_{1}$ distinct from $\beta$ satisfies $|\beta| \geq\left|\gamma_{1}\right| M^{\prime}$ we are done. Thus assume for any such separating $\Gamma_{1},\left|\gamma_{1}\right| \geq|\beta| / M^{\prime}$. Since $|\beta| \geq\left|\Omega_{0}\right| / M$ we have $\left|\gamma_{1}\right| \geq\left|\Omega_{0}\right| / M M^{\prime}$. Suppose inductively we have constructed $\beta=\Gamma_{0}, \Gamma_{1}, \ldots, \Gamma_{k}, M_{i}=M_{i}\left(M, M^{\prime}\right)$, and $D_{i}, i=0, \ldots, k$ such that

(i) $\Gamma_{i+1}$ properly separates $\Gamma_{i}$ from $\Omega_{0}-\beta$.

(ii) $\left|\Gamma_{i}-\Gamma_{i-1}\right| \leq M^{\prime}\left|\Gamma_{i+1}-\Gamma_{i}\right|$.

(iii) the longest curve $\gamma_{i} \in \Gamma_{i}-\Gamma_{i-1}$ is $D_{i}$ close to $\Gamma_{i+1}-\Gamma_{i}$.

(iv) $\left|\gamma_{i+1}\right| \geq\left|\Omega_{0}\right| / M_{i}$.

We have given the construction for $k=1$. Suppose $\gamma_{k}$ is 2-close to $\Omega_{0}-\Gamma_{k}$. Then by Lemma 1.1, applied with $\alpha=\gamma_{k-1}, \beta=\gamma_{k}, \gamma \in \Omega_{0}-\Gamma_{k}$, and (ii), (iii), and (iv) above we have that $\gamma_{k-1}$ is $D=D_{k-1} M_{k-1}+2 M^{\prime}$-close to $\Omega_{0}-\Gamma_{k}$.

Then since $\Omega_{0}-\Gamma_{k-1} \subseteq\left(\Omega_{0}-\Gamma_{k}\right) \cup\left(\Gamma_{k}-\Gamma_{k-1}\right)$, and $\gamma_{k-1}$ is $D$-close to both $\Omega_{0}-\Gamma_{k}$ and $\Gamma_{k}-\Gamma_{k-1}$ it is $D$-close to $\Omega_{0}-\Gamma_{k-1}$. Repeating this argument with the hypothesis $\gamma_{k-1} D$-close to $\Omega_{0}-\Gamma_{k-1}$ replacing $\gamma_{k} D=2$-close to $\Omega_{0}-\Gamma_{k}$, we find eventually that $\beta D$-close to $\Omega_{0}-\beta$ to begin with.

Thus we may assume $\gamma_{k}$ is not 2-close to $\Omega_{0}-\Gamma_{k}$. From $\Gamma_{k}$ we wish to either construct a new sequence $\beta=\Gamma_{0}, \ldots, \Gamma_{\text {l }}$ satisfying (i)-(iv) and such that $\Gamma_{\text {l }}$ properly separates $\Gamma_{k}$ from $\Omega_{0}-\beta$ or to simply find the $\Omega_{0}^{\prime}$ that we desired. To do that we add a triangle with $\gamma_{k}$ as one side arriving at $\Gamma_{k+1}$ separating $\Gamma_{k}$ from $\Omega_{0}-\beta$ with $\gamma_{k}$ 2-close to $\Gamma_{k+1}-\Gamma_{k}$. 
If now $\left|\gamma_{k}\right| \leq M^{\prime}\left|\Gamma_{k+1}-\Gamma_{k}\right|$ then (ii) and (iv) are satisfied as well as (i) and (iii) for the sequence $\Gamma_{0}, \ldots, \Gamma_{k}, \Gamma_{k+1}$.

If, on the other hand, $\left|\gamma_{k}\right| \geq M^{\prime}\left|\Gamma_{k+1}-\Gamma_{k}\right|$, then again by applying Lemma 1.1, with $\alpha=\gamma_{k-1}, \beta=\gamma_{k}, \gamma \in \Gamma_{k+1}-\Gamma_{k-1}$, the assumption $\left|\gamma_{k}\right| \geq M^{\prime}\left|\Gamma_{k+1}-\Gamma_{k}\right|$ and (ii), (iii), give

$$
\gamma_{k-1} \quad D=D M^{\prime}+2 M^{\prime} \text {-close to } \Gamma_{k+1}-\Gamma_{k-1} .
$$

If now $\left|\gamma_{k-1}\right| \leq M^{\prime}\left|\Gamma_{k+1}-\Gamma_{k-1}\right|$, then applying Lemma 1.1 again using $\alpha=\gamma_{k-2}$, $\beta=\gamma_{k-1}$ and $\gamma \in \Gamma_{k+1}-\Gamma_{k-1}$ we find $\gamma_{k-2} D$-close to $\Gamma_{k+1}-\Gamma_{k-1}$. Since $\Gamma_{k+2}-\Gamma_{k-1} \subset$ $\left(\Gamma_{k+1}-\Gamma_{k-1}\right) \cup\left(\Gamma_{k-1}-\Gamma_{k-2}\right), \gamma_{k-2}$ is $D$-close to $\Gamma_{k+2}-\Gamma_{k-1}$. If $\left|\Gamma_{k+2}-\Gamma_{k-2}\right| \leq$ $\left|\gamma_{k-2}\right| / M^{\prime}$ then $\gamma_{k-3} D$-close to $\Gamma_{k+1}-\Gamma_{k-2}$. Either we can continue in this manner for all $k$; we have $\beta$ is $D$-close to $\Gamma_{k+1}$ and we have found the $\Omega_{0}^{\prime}=\Gamma_{k+1}$ that we desired, or there is a $\Gamma_{j}$ such that

$$
\left|\Gamma_{k+1}-\Gamma_{j}\right| \geq\left|\gamma_{j}\right| / M^{\prime} .
$$

In that case we will have constructed a sequence $\Gamma_{0}, \Gamma_{1}, \ldots, \Gamma_{j}, \Gamma_{k+1}$ relabelled $\Gamma_{0}, \ldots, \Gamma_{l}$ satisfying (i)-(iv). Thus either there is an $\Omega_{0}^{\prime}$ or we have found a new sequence. If the latter, there must be a maximal $\Gamma_{p}$ constructed after at most $C_{1}$ steps; namely a sequence $\Gamma_{0}, \ldots, \Gamma_{p}$ satisfying (i)-(iv) such that there is no sequence $\Gamma_{0}, \ldots, \Gamma_{l}$ satisfying (i)-(iv) such that $\Gamma_{l}$ separates $\Gamma_{p}$ from $\Omega_{0}-\beta$. We will use this maximal sequence to find $\Omega_{0}^{\prime}$.

Now if $\gamma_{p}$ is 2-close to $\Omega_{0}-\Gamma_{p}$, then just as in the first paragraph of the proof, Lemma 1.1 (ii), (iii) and (iv) show $\beta D$-close to $\Omega_{0}$ to begin. Thus we may assume $\gamma_{p}$ is not 2-close to $\Omega_{0}-\Gamma_{\mathrm{p}}$. Then construct $\Gamma_{p+1}$ separating $\Gamma_{\mathrm{p}}$ from $\Omega_{0}$ just as we constructed $\Gamma_{k+1}$ from $\Gamma_{k}$. We must have $\left|\Gamma_{p+1}-\Gamma_{p}\right| \leq\left|\gamma_{p}\right| / M^{\prime}=\left|\Gamma_{p}-\Gamma_{p-1}\right| / M^{\prime}$ for otherwise just as in the third paragraph $\Gamma_{0}, \ldots, \Gamma_{p+1}$ would be a new sequence satisfying (i)-(iv) contradicting the maximality of $\Gamma_{p}$. Then by (ii), (iii), (iv) and Lemma 1.1, $\gamma_{p-1} D$-close to $\Gamma_{p+1}-\Gamma_{p-1}$. Again $\left|\Gamma_{p+1}-\Gamma_{p-1}\right| \leq\left|\gamma_{p-1}\right| / M^{\prime}$ for otherwise $\Gamma_{0}, \ldots, \Gamma_{p-1}, \Gamma_{p+1}$ would satisfy (i)-(iv), contradicting $\Gamma_{p}$ maximal. This implies $\gamma_{p-2} D$-close to $\Gamma_{p+1}-\Gamma_{p-2}$. Continuing, we find that $\beta$ is $D$-close to $\Gamma_{p+1}$ and we let $\Omega_{0}^{\prime}=\Gamma_{p+1}$.

Proof of Proposition 1.6. We let $M^{\prime}=\left(2 C_{1}\right)^{2 C_{1}}$. Since $\beta$ is not $M$-separated from $\alpha$ we have $|\beta| \geq|\alpha| / M$. We assume $\beta$ is not $D=D\left(M, M^{\prime}, C_{1}\right)$-close to $\alpha, D$ given by Proposition 1.7. We will construct a sequence of disjoint systems $\beta=\Omega_{0}, \Omega_{1}, \ldots, \Omega_{n}=\alpha$ such that for $\iota \geq 1, \Omega_{i}$ separates $\Omega_{i-1}$ from $\alpha$ and for each $\omega_{i} \in \Omega_{i}-\Omega_{i-1}$ there is a $\omega_{i-1} \in \Omega_{i-1}-\Omega_{i}$ such that $\omega_{i-1}$ is $D$-close to $\omega_{i}$ and not $M_{1}$-separated from $\omega_{i}$.

We begin by constructing $\Omega_{1}$. By Proposition 1.7 there is a $\Gamma_{1}$ properly separating $\beta$ from $\alpha$ such that

$$
|\beta| \geq M^{\prime}\left|\Gamma_{1}\right| \text { and } \beta \quad D \text {-close to } \Gamma_{1} .
$$

We may assume there is no $\Gamma$ properly separating $\beta$ from $\gamma_{1}$ with the same properties.

If $\Gamma$ is any system property separating $\beta$ from $\Gamma_{1}$ and $|\beta| \geq|\Gamma| / M$ then $\beta$ is $D$-close to $\Gamma$. For if not, then again by Proposition $1.7, \beta$ could be properly separated from 
$\Gamma$ by $\Gamma^{\prime}$ such that $|\beta| \geq M^{\prime}\left|\Gamma^{\prime}\right|$ and $\beta D$-close to $\Gamma^{\prime}$. But then $\Gamma^{\prime}$ also properly separates $\beta$ from $\Gamma_{1}$ and this contradicts the choice of $\Gamma_{1}$. Thus if there is some $\Gamma$ properly separating $\beta$ from $\Gamma_{1}$ with $|\Gamma| \leq 2 C_{1}\left|\Gamma_{1}\right| \leq|\beta|$, replace $\Gamma_{1}$ with $\Gamma$ and $\beta$ is still $D$-close to $\Gamma$. If there is a further $\Gamma^{\prime}$ properly separating $\beta$ from $\Gamma$ with $\left|\Gamma^{\prime}\right| \leq 2 C_{2}|\Gamma| \leq|\beta|$ then we replace $\Gamma$ with $\Gamma^{\prime}$. We can do this at most $C_{1}$ times. By the choice of $M^{\prime}$ we eventually find $\Omega_{1}$ properly separating $\beta$ from $\Gamma_{1}$ such that

(i) $|\beta| \geq\left(M^{\prime} /\left(2 C_{1}\right)^{\left.C_{1}\right)}\right)\left|\Omega_{1}\right|$

(ii) $\beta D$-close to $\Omega_{1}$

(iii) there is no $\Gamma$ properly separating $\beta$ from $\Omega_{1}$ with $|\Gamma| \leq 2 C_{1}\left|\Omega_{\mathrm{l}}\right|$.

We claim this implies

(iv) $\beta$ is not $C_{1}$-separated by $\Gamma^{\prime}$ from any $\omega_{1} \in \Omega_{1}$.

For if there were such an $\omega_{1}$, we could combine $\Gamma^{\prime}$ and $\Omega_{1}$ using Proposition 1.3 to find $\Gamma$ separating $\beta$ from $\Gamma_{1}$ such that

$$
|\Gamma| \leq C_{1}\left(\left|\Gamma^{\prime}\right|+\left|\Omega_{1}\right|\right) \leq C_{1}\left(\frac{\left|\Omega_{1}\right|}{C_{1}}+\left|\Omega_{1}\right|\right) \leq 2 C_{1}\left|\Omega_{1}\right|<|\beta| .
$$

Then $\beta \notin \Gamma$ so $\Gamma$ properly separates $\beta$ from $\Omega_{1}$, a contradiction. Since each $\omega \in \Omega_{1}$ satisfies $|\omega| \leq|\beta|$ and each is $D$-close to $\beta$, Lemma 1.1 applied with $\alpha=\omega, \gamma=\omega^{\prime} \in \Omega_{1}$ gives

(v) $\omega^{\prime}, \omega \in \Omega_{1}$ are $D+D=2 D$-close to each other.

Since $\Omega_{1}$ separates $\beta$ from $\alpha$, by assumption,

(vi) $\left|\Omega_{1}\right| \geq|\alpha| / M$.

We also claim

(vii) Some $\omega \in \Omega_{1}$ is not $M M^{\prime}$-separated from $\alpha$. For if all $\omega \in \Omega_{1}$ were so separated, we could combine at most $C_{1}$ such separating $\Gamma$, using Corollary 1.4 , to find $\Gamma, M$-separating $\Omega_{1}$ from $\alpha$. But this $\Gamma$ also separates $\beta$ from $\alpha$ contradicting (vi).

Suppose the longest saddle $\omega_{1} \in \Omega_{1}$ is $D$-close to $\alpha$. Then by Lemma 1.1 applied to any $\omega \in \Omega_{1}, \omega_{1}$, and $\alpha$ and (v) above we have for all $\omega \in \Omega_{1}$,

$$
\omega \quad D^{\prime}=2 D M+D \text {-close to } \alpha \text {. }
$$

Choose by (vii) some $\omega$ not $M_{1}=M M^{\prime}$-separated from $\alpha$. Then by (ii) $\beta$ is $D$-close to $\omega$ and by (iv) is not $C_{1}$-separated from it. Our desired sequence is $\beta, \omega, \alpha$.

Suppose on the other hand this longest $\omega_{1}$ is not $D$-close to $\alpha$. We wish to construct $\Omega_{2}$. Choose $\Omega_{2}^{\prime}$ to have the same properties (i)-(iv) with respect to $\omega_{1}$ and $\alpha$ that $\Omega_{1}$ had with respect to $\beta$ and $\alpha$. In particular it properly separates $\omega_{1}$ from $\alpha$.

Case I. If $\Omega_{2}^{\prime}$ separates all of $\Omega_{1}$, not just $\omega_{1}$, from $\alpha$, let $\Omega_{2}=\Omega_{2}^{\prime}$. Since $\omega_{1}$ is $D$-close to $\Omega_{2}$, by Lemma 1.1 and (v) each $\omega \in \Omega_{1}$ is $D+2 D=3 D$-close to $\Omega_{2}$.

Case II. If $\Omega_{2}^{\prime}$ does not separate all of $\Omega_{1}$ from $\alpha$, let $\Omega_{2}^{\prime \prime}$ be the combination of $\Omega_{1}$ and $\Omega_{2}^{\prime}$. Since $\omega_{1}$ is $2 D$-close to $\Omega_{1}$ and $D$-close to $\Omega_{2}$ it is $3 C_{1} D$-close to $\Omega_{2}^{\prime \prime}$ by Proposition 1.3(i). Moreover $\left|\Omega_{2}^{\prime \prime}\right| \leq C_{1}\left(\left|\Omega_{1}\right|+\left|\Omega_{2}^{\prime}\right|\right) \leq 2 C_{1}\left|\Omega_{1}\right|$. These two facts again with Lemma 1.1 show each $\omega \in \Omega_{1}$ is $2 D 2 C_{1}+3 D C_{1}=7 D C_{1}$-close to $\Omega_{2}^{\prime \prime}$. Moreover it is clear any two $\omega \in \Omega_{2}^{\prime \prime}$ are $D^{\prime}$-close to each other for some $D^{\prime}$ just as all $\omega \in \Omega_{1}$ are $2 D$-close to each other. Let $V$ be the component of the complement of $\Omega_{2}^{\prime \prime}$ that 
contains $\alpha$. Finally, let

$$
\Omega_{2}=\Omega_{2}^{\prime \prime} \cap V .
$$

In either case we have constructed $\Omega_{2}$ such that each $\omega \in \Omega_{1}$ is $D$ close to $\Omega_{2}$ for some $D$. We need to show for each $\omega_{2} \in \Omega_{2}-\Omega_{1}$ there is a $\omega \in \Omega_{1}$ not $M_{1}$-separated from it for some $M_{1}, M_{1}$ universal.

In the first case where $\Omega_{2}=\Omega_{2}^{\prime}$ we have by construction property (iv): namely $\omega_{1}$ is not $C_{1}$-separated from $\omega_{2}$.

The second case is more complicated. One subcase is already taken care of. If $\omega_{2} \in \Omega_{2}-\Omega_{1}$ is already a saddle connection of $\Omega_{2}^{\prime}$, then just as above, $\omega_{1}$ cannot be $C_{1}$ separated from it. Therefore assume $\omega_{2}$ is not a saddle connection of $\Omega_{2}^{\prime}$. The reason this case is more difficult is that since $\omega_{2}$ is formed from pieces of $\Omega_{1}$ and $\Omega_{2}^{\prime}$ it may be much longer than $\Omega_{2}^{\prime}$. Thus a $\Gamma$ separating $\omega_{1}$ from it may also be longer than $\Omega_{2}^{\prime}$ so the combination of $\Gamma$ and $\Omega_{2}^{\prime}$ does not give a contradiction to (iv).

Now $\omega_{2}$ is homotopic to a finite alternating sequence $\sigma=\cdots \omega * \omega^{\prime} \cdots$ of arcs $\omega$ of $\Omega_{1}$ and $\omega^{\prime}$ of $\Omega_{2}^{\prime}$. Together $\omega_{2}$ and $\sigma$ bound a region $Z$ which is either simply connected or an annulus. Now suppose each $\omega \in \Omega_{1}$ which has a subarc appearing in $\sigma$ is $M_{1}$-separated from $\omega_{2}$ by some $\Gamma$. Then it is easy to see $\omega_{2}$ is homotopic to a union of subarcs of $\Gamma \cap Z$ and subarcs of the $\omega^{\prime} \cap Z$. Now $\omega_{2}$ is a geodesic and thus shorter than this union. Thus

$$
\left|\omega_{2}\right| \leq \sum_{\omega^{\prime} \in \Omega_{2}^{\prime}}\left|\omega^{\prime} \cap Z\right|+\sum_{\gamma \in \Gamma}|\gamma \cap Z| \leq C_{1}\left(\left|\Omega_{2}^{\prime}\right|+\left|\omega_{2}\right| / M_{1}\right) .
$$

For $M_{1} \geq 2 C_{1}$ this implies

$$
\left|\omega_{2}\right| \leq 2 C_{1}\left|\Omega_{2}^{\prime}\right| \text {. }
$$

Next suppose some $\Gamma^{\prime} 2 C_{1}$-separates $\omega_{1}$ from $\omega_{2}$. Then the above inequality gives $\left|\Gamma^{\prime}\right| \leq\left|\Omega_{2}^{\prime}\right|$. We can't have $\Gamma^{\prime}$ separating $\omega_{1}$ from $\Omega_{2}^{\prime}$ for this would contradict the definition of $\Omega_{2}^{\prime}$. Nor can $\Gamma^{\prime}$ intersect the component of the complement of $\Omega_{2}^{\prime}$ containing $\omega_{1}$. For then the combination $\Gamma$ of $\Gamma^{\prime}$ and $\Omega_{2}^{\prime}$ would separate $\omega_{1}$ from $\Omega_{2}^{\prime}$ and still satisfy

$$
|\Gamma| \leq 2 C_{1}\left|\Omega_{2}^{\prime}\right|
$$

still a contradiction. Thus in fact $\Omega_{2}^{\prime}$ must properly separate $\omega_{1}$ from $\Gamma^{\prime}$. Since $\Gamma^{\prime}$ separates $\omega_{1}$ from $\omega_{2}$ it must also separate $\Omega_{2}^{\prime}$ from $\omega_{2}$. Otherwise a path from $\Omega_{2}^{\prime}$ to $\omega_{2}$ missing $\Gamma^{\prime}$ could be connected to a path joining $\Omega_{2}^{\prime}$ to $\omega_{1}$ giving a path from $\omega_{1}$ to $\omega_{2}$ missing $\Gamma^{\prime}$.

Then since $\Gamma^{\prime}$ separates $\Omega_{2}^{\prime}$ from $\omega_{2}, \Gamma^{\prime} \cap Z$ separates $\Omega_{2}^{\prime} \cap Z$ from $\omega_{2}$ in $Z$. Recall previously we have subarcs of $\Gamma \cap Z$ together with subarcs of $\Omega_{2}^{\prime} \cap Z$ homotopic to $\omega_{2}$. Now we must have a union of subarcs of $\Gamma \cap Z$ and subarcs of $\Gamma^{\prime} \cap Z$ homotopic to $\omega_{2}$.

But the sum of lengths of subarcs of $\Gamma \cap Z \leq C_{1}|\Gamma|$ and sum of length of subarcs of $\Gamma^{\prime} \cap Z \leq C_{1}\left|\Gamma^{\prime}\right|$. Since $\omega_{2}$ is a geodesic

$$
\left|\omega_{2}\right| \leq C_{1}|\Gamma|+C_{1}\left|\Gamma^{\prime}\right| \leq \frac{C_{1}\left|\omega_{2}\right|}{M_{1}}+\frac{C_{1}}{2 C_{1}}\left|\omega_{2}\right| .
$$


For $M_{1}>2 C_{1}$ this is a contradiction. We have shown for each $\omega_{2} \in \Omega_{2}$ there is some $\omega \in \Omega_{1}$ not $M_{1}$-separated from it. We now repeat the argument with $\Omega_{2}$ in place of $\Omega_{1}$, if necessary find $\Omega_{3}$. After at most $C_{1}$ steps we have our desired sequence of $\Omega_{i}$.

The next construction associates to every short saddle connection a complex with boundary that is 'isolated' in a certain sense. This construction will be used to deal with the problem of saddle connections that are not $\varepsilon$-wide.

We first fix two additional constants for the rest of the paper. Others will be fixed as we go along. Fix $C>1$ and $0<\sigma<1$. We require

$$
C>\max \left(\frac{16}{\sigma}, 2 C_{1}-1\right) \text {. }
$$

Also in the rest of the paper $M$ with subscripts will also refer to absolute constants depending only on the above constants.

Definition. An $\varepsilon^{\prime}$ subcomplex is a triangulation of a subset of $Y$ such that the vertices are zeroes of $\varphi$ and the edges are saddle connections of length at most $\varepsilon^{\prime}$ and the faces are triangles without zeroes in their interior. We assume if three edges of a subcomplex bound a triangle, it is included in the subcomplex. A subcomplex has an interior if it contains a face.

Definition. A boundary edge is an edge which bounds less than two triangles in the complex.

LEMMA 1.8. The maximal area of an $\varepsilon^{\prime}$ complex is

$$
A\left(\varepsilon^{\prime}\right)=\frac{3^{1 / 2} \varepsilon^{\prime 2}}{6} C_{1} .
$$

If $A\left(\varepsilon^{\prime}\right)<1$, an $\varepsilon^{\prime}$ complex has a boundary edge.

Proof. The maximal area of a triangle whose sides are length $\leq \varepsilon^{\prime}$ is $\varepsilon^{\prime 2} 3^{1 / 2} / 4$. There are at most $2 C_{1} / 3$ triangles in a complex.

Definition. $\beta$ is $\left(M, \varepsilon^{\prime}\right)$-isolated if $|\beta| \leq M \varepsilon^{\prime}$ and if any $\gamma$ crossing $\beta$ satisfies $|\gamma| \geq C \max \left(|\beta|, \varepsilon^{\prime}\right)$.

Definition. A system $\Gamma$ is $\left(M, \varepsilon^{\prime}\right)$-isolated if $|\Gamma| \leq M \varepsilon^{\prime}$ and for every $\gamma$ crossing $\Gamma$, $|\gamma| \geq C \max \left(|\Gamma|, \varepsilon^{\prime}\right)$. Notice that isolated saddle connections cannot cross since $C>1$. We now have the following basic construction.

Lemma 1.9. [K-M-S]. Suppose $X$ is a connected complex and the boundary $\Gamma$ is not $\left(1, \varepsilon^{\prime}\right)$ isolated. Then there is a connected $\varepsilon^{\prime}+C \varepsilon^{\prime}$ complex. $X_{1} \supset X$ with more edges and triangles.

We need a slightly more general construction. Let $\Gamma$ be a system of saddle connections and $U$ a complementary component.

Definition. An $\varepsilon$-extension of the pair $(\Gamma, U)$ consists of a complex $X_{1} \subset \bar{U}$ with nonempty interior such that the boundary $\Gamma^{\prime}$ satisfies

(i) $\left|\Gamma^{\prime}\right| \leq(C+1)^{d} \varepsilon$ 
(ii) area $X_{1} \leq A\left(\varepsilon(C+1)^{d}\right)$ where $d$ is the number of edges needed to triangulate $U$.

LeMMA 1.10. Suppose $X$ is an $\varepsilon$ complex with boundary $\Gamma$ as in Lemma 1.9. Then for some component of the complement $U$ of $X,\left(X_{1}-X\right) \cap U$ is an E-extension of $(\Gamma, U)$.

Proof. Since $X_{1}$ is a $(C+1) \varepsilon$ complex, area $\left(X_{1}-X\right) \leq A((C+1) \varepsilon)$. For some complementary $U,\left(X_{1}-X\right) \cap \bar{U}$ has nonempty interior and its boundary has length $\leq(C+1) \varepsilon$.

The main construction now is the following:

Proposition 1.11. There are constants $C_{2}, C_{3}, M$ such that for any $\delta>0$ there is an $\varepsilon>0$ such that for all systems $\Gamma_{0}$ dividing the surface into one or two components, if $\varepsilon_{1}=\left|\Gamma_{0}\right| \leq \varepsilon$ and $\Gamma_{0}$ is not $\left(1,\left|\Gamma_{0}\right|\right)$ isolated, then for each complementary component $U$ which has area $\geq \delta$ there is a domain $U_{1} \nsubseteq U$ with boundary $\Gamma_{1}$ such that

(a) $\Gamma_{1}$ separates $\Gamma_{0}$ from $U_{1}$,

(b) $\Gamma_{0} \not \subset \Gamma_{1}$,

(c) area $U_{1} \geq g(\varepsilon, \delta)=(\delta / M)-f(\varepsilon)$ where $\lim _{\varepsilon \rightarrow 0} f(\varepsilon)=0$,

(d) $\left|\Gamma_{1}\right| \leq C_{2} \varepsilon_{1} \leq C_{2} \varepsilon$,

(e) $\Gamma_{1}$ is $\left(C_{2}, \varepsilon_{1}\right)$-isolated,

(f) $\left(\Gamma_{1}, U_{1}\right)$ cannot be $C_{2} \varepsilon_{1}$-extended,

(g) there is no $\Gamma C_{3}$-separating $\Gamma_{0}$ from $\Gamma_{1}$,

(h) $\Gamma_{1}$ is minimal with respect to $(\mathrm{a})-(\mathrm{f})$ in the sense that there is no $\Gamma^{\prime} \subset \bar{U}$ properly separating $\Gamma_{0}$ from $\Gamma_{1}$ also satisfying $(\mathrm{a})-(\mathrm{g})$.

Remark. By Lemma 1.10, (e) follows from (f) but we include it for emphasis. The constants $C_{2}, C_{3}$ will be fixed for the rest of the paper.

Proof. Let $\varepsilon_{1}=\left|\Gamma_{0}\right|$. By assumption, $\Gamma_{0}$ is crossed by saddles of length $\leq C \varepsilon_{1}$. By Lemmas 1.9 and 1.10 there is an $\varepsilon_{1}$-extension $X_{1}$ of $\left(\Gamma_{0}, U\right)$. Area $X_{1} \leq(C+1)^{C_{1}} \varepsilon_{1}$. For $\varepsilon$ sufficiently small $X_{1}$ is a proper subset of $U$. $U-X_{1}$ has at most $C_{1}$ components. Thus there is a component $V_{1}$ of $U-X_{1}$ with area $V_{1} \geq\left(\delta /(C+1)^{C_{1}} \varepsilon_{1}\right) / C_{1}$. Moreover the boundary $\Gamma^{1}$ of $V_{1}$ satisfies $\left|\Gamma_{1}\right| \leq(C+1)^{C_{1}} \varepsilon_{1}$.

Let $\varepsilon_{2}=\left(C_{1}+1\right)^{C_{1}} \varepsilon_{1}$. Again for $\varepsilon$ sufficiently small, if $\left(\Gamma^{1}, V_{1}\right)$ can be $\varepsilon_{2}$-extended to $X_{2}$, then $X_{2}$ is a proper subset of $V_{1}$ and some component $V_{2}$ of $V_{1}-X_{2}$ has area

$$
\geq \frac{\text { area } V_{1}-(C+1)^{C_{1}} \varepsilon_{2}}{C_{1}} \text {. }
$$

The boundary $\Gamma^{2}$ of $V_{2}$ satisfies $\left|\Gamma_{2}\right| \leq(C+1)^{C_{1}} \varepsilon_{2}$. Since there are at most $p \leq C_{1}$ steps in this process, there are constants $M, C_{2}$ and a function $f(\varepsilon)$ such that there must be $U_{1} \subset U$ with boundary $\Gamma_{1}$ such that area

$$
U_{1} \geq \frac{\delta}{M}-f\left(\varepsilon_{1}\right)
$$

$\left|\Gamma_{1}\right| \leq C_{2} \varepsilon_{1}$ and such that $\left(\Gamma_{1}, U_{1}\right)$ cannot be $C_{2} \varepsilon_{1}$-extended. Thus by construction (a), (b) are satisfied as well as (c), (d), (f). As remarked before, (e) is satisfied by Lemma 1.10. Let $X\left(\Gamma_{0}\right)=X_{1} \cup X_{2} \cup \cdots \cup X_{p}$ the complex constructed. Since some $\left(\Gamma_{1}, U_{1}\right)$ exists that satisfies (a)-(f) we can always find a minimal one. Now we 
claim for some $C_{3},(\mathrm{~g})$ is satisfied as well. Suppose $\Gamma C_{3}$-separates $\Gamma_{0}$ from $\Gamma_{1}$. Let $\varepsilon^{\prime}=|\Gamma| \leq\left|\Gamma_{1}\right| / C_{3}$. Let $V \subset U$ be the component of the complement of $\Gamma$ containing $U_{1}$ so area $V \geq$ area $U_{1}$.

If $C_{3}$ is sufficiently large, $\varepsilon^{\prime}<\varepsilon_{1}$. If $(\Gamma, V)$ cannot be $\varepsilon^{\prime}$-extended we have already contradicted $\Gamma_{1}$ minimal. If $(\Gamma, V)$ can be $\varepsilon^{\prime}$-extended then the process described above for $\left(\Gamma_{0}, U\right)$ applied instead to $(\Gamma, V)$ will lead to $\Gamma^{*}, V^{*} \subset V$ and $X^{*}(\Gamma)$ such that $\left(\Gamma^{*}, V^{*}\right)$ cannot be $C_{2} \varepsilon^{\prime}$-extended, where again for $C_{3}$ sufficiently large,

$$
\text { area } V^{*} \geq \frac{\delta}{M}-f\left(\varepsilon^{\prime}\right),\left|\Gamma^{*}\right| \leq C_{2} \varepsilon^{\prime} \leq C_{2} \varepsilon_{1} \text {. }
$$

Now we claim $\Gamma^{*}$ must separate $\Gamma$ from $\Gamma_{1}$. To prove the claim, notice first $\Gamma^{*}$ cannot intersect $\Gamma_{1}$ since $\Gamma_{1}$ is $C_{2} \varepsilon_{1}$-isolated. Thus $\Gamma_{1}$ and $\Gamma^{*}$ are disjoint. Nor can $\Gamma^{*}$ intersect $U_{1}$ for then $X^{*}(\Gamma) \cap U_{1} \neq \varnothing$ and $\left(\Gamma_{1}, U_{1}\right)$ could be $C_{2} \varepsilon_{1}$-extended to $X^{*}(\Gamma) \cap U_{1}$, a contradiction. Thus $\Gamma^{*}$ must separate $\Gamma$ from $\Gamma_{1}$, hence also $\Gamma_{0}$ from $\Gamma_{1}$, proving the claim. But then $\left(\Gamma_{1}, U_{1}\right)$ is not minimal and we have a contradiction, proving the Proposition.

Now we will choose $\varepsilon$ which will be fixed for the rest of the paper. For each $\varepsilon$ let

$$
g(\delta)=\frac{\delta}{M}-f\left(4 C_{2} \varepsilon\right)
$$

be the function given in Proposition 1.11(c). Given $\delta_{0}=\frac{1}{2}$ let $\delta_{1}, \ldots, \delta_{C_{1}}$ be the values of the $C_{1}$ iterates of $g$ evaluated at $\delta_{0}=\frac{1}{2}$. Since $\lim _{\varepsilon \rightarrow 0} f(\varepsilon)=0, \varepsilon$ can be chosen so $\delta_{1}, \ldots, \delta_{C_{1}}$ are bounded below away from zero. Choose $\varepsilon$ sufficiently small so that for each $\delta_{i}$, the value of $4 C_{2} \varepsilon$ is such that Proposition 1.11 holds.

Now let $C_{4}=2 C_{3}\left(2 C_{1}\right)^{C_{1}}$. The constant $C_{4}$ will be fixed for the rest of the paper.

Corollary 1.12. Suppose $\Gamma_{0}, \Gamma_{1}$ are as in Proposition 1.11. Then there is a $\gamma_{0} \in \Gamma_{0}$ not $C_{4}$-separated from $\Gamma_{1}$.

Proof. If every $\gamma_{0}$ in $\Gamma_{0}$ were $C_{4}$-separated from $\Gamma_{1}$, we could combine all separating $\Gamma$ using Corollary 1.4 to find $\Gamma$ separating all of $\Gamma_{0}$ from $\Gamma_{1}$. Such a $\Gamma$ gives

$$
|\Gamma| \leq\left(2 C_{1}\right)^{C_{1}}\left|\Gamma_{1}\right| / C_{4} \leq\left|\Gamma_{1}\right| / C_{3},
$$

a contradiction to the conclusion of Proposition 1.11.

\section{The number of isolated saddle connections}

In this section we will compute the number of systems of saddle connections that simultaneously become isolated within a certain minimal period of time. In particular this will include the set of saddle connections mentioned in the introduction. Recall now we have fixed $\varepsilon, C_{2}$. We will refer to $\left(C_{2}, \varepsilon\right)$ isolated system simply as isolated. We will also simply write $\beta$ is $\varepsilon^{\prime}$-isolated to refer to $\beta$ as $\left(1, \varepsilon^{\prime}\right)$-isolated.

Definition. A system $\Gamma$ on $Y$ is $\varepsilon$-wide if either

(i) $|\Gamma| \leq 4 C_{2} \varepsilon$ and $\Gamma$ is isolated, or

(ii) $|\Gamma| \geq 4 C_{2} \varepsilon$ and for some $(\theta, t)|\Gamma| \leq \varepsilon$ and is isolated with respect to $\varphi_{\theta, r}$ on $Y_{\theta, 1}$ and $v_{t, 1}(\Gamma) \geq \varepsilon / C_{3}$. 
Remark. This last condition in (ii) is essential for Propositions 2.1 and 2.2. The point is the following. A saddle connection may become short after a certain time if the vertical length becomes small and the horizontal length remains small. It may then remain small for a later time if the horizontal length remains small since the vertical length continues to decrease. The condition of $v_{\theta, t}(\Gamma)$ says that up to a factor $C_{3}$ the time $t$ is as small as possible to make $|\Gamma| \leq \varepsilon$.

Example. Suppose $\Gamma$ is a system that is the boundary of an annulus of width $\geq C \varepsilon^{2} /|\Gamma|$ swept out by closed trajectories of length $|\Gamma|$. Let $\theta=\theta_{\Gamma}$ be the angle so $v_{\theta_{\Gamma}}(\gamma)=|\gamma|$ for $\gamma \in \Gamma$ and let $e^{t / 2}=|\Gamma| / \varepsilon$. Then $|\Gamma|_{\theta, t}=\varepsilon$. Any curve crossing $\Gamma$ crosses the annulus. The annulus has width $\geq C \varepsilon$ with respect to $\varphi_{\theta, t}$ so $\Gamma$ is isolated at time $t$ and thus $\varepsilon$-wide on the base surface.

Example. If the width $<C \varepsilon^{2} /|\Gamma|, \Gamma$ may not be $\varepsilon$-wide for any $(\theta, t)$.

We now define several sets of saddle connections. Let

$$
\begin{aligned}
S(n) & =\{\text { saddle connections on } X \text { of length } \leq n\} \\
S_{\sigma}(n) & =\{\beta \in S(n): \sigma n \leq|\beta| \leq n\} \\
S^{\varepsilon}(n) & =\{\beta \in S(n): \beta \text { is } \varepsilon \text {-wide }\} .
\end{aligned}
$$

Note. Theorem 1 is equivalent to showing card $S_{\sigma}(n)$ is $0\left(n^{2}\right)$.

Now suppose $\alpha$ is a saddle connection on $Y$. We need to count saddle connections disjoint from $\alpha$. Let

$$
\begin{aligned}
T(n, \alpha) & =\{\text { saddle connections } \beta \text { disjoint from } \alpha:|\beta| \leq n|\alpha|\} \\
T(n, \alpha, M) & =\{\beta \in T(n, \alpha): \beta \text { is not } M \text {-separated from } \alpha\} \\
T^{\varepsilon}(n, \alpha, D) & =\{\beta \in T(n, \alpha): \beta \text { is } D \text {-close to } \alpha \text { and } \varepsilon \text {-wide }\} \\
T(n, \alpha, M, D) & =\{\beta \in T(n, \alpha, M): \beta \text { is } D \text {-close to } \alpha\}
\end{aligned}
$$

Our main result about these latter sets is

THEOREM 4.1. card $T(n, \alpha, M)$ is $0\left(n(\log n)^{k}\right)$ for some $k$, and bound 0 depending on $M, C_{1}$ but not $n$ or $|\alpha|$.

Remark. The importance of this result, proved in $\S 4$, is that the cardinality depends only on the ratio $n$, of the length of $\beta$ to the length of $\alpha$, and is less than quadratic in that ratio.

We first give the computations for $\varepsilon$-wide saddle connections.

Proposition 2.1. card $S^{\varepsilon}(n)$ is $0\left(n^{2}\right)$.

Proposition 2.2. card $T^{f}(n, \alpha, D)$ is $0(n)$.

Remark. The bounds depend on $C_{1}, \varepsilon$ and in Proposition 2.2 on $D$ as well but not on $|\alpha|$ or $n$ or on the quadratic differential in either case.

The propositions are based on two simple computations which we state as lemmas. The first says that if $|\beta|_{\theta, 1}$ is small, $\theta$ is near the vertical angle $\theta_{\beta}$. The second says that if two saddle connections are isolated at a certain time and they cross, their vertical angles can not be too close to each other. For the lemmas assume

$$
|\beta| \geq 4 C_{2} \varepsilon \text { and }|\beta|_{H_{1}} \leq \varepsilon^{\prime} \leq C_{2} \varepsilon .
$$

LEMMA 2.3. $\left|\theta-\theta_{\beta}\right| / 2 \leq 2 \varepsilon^{\prime} \mathrm{e}^{-1 / 2} /|\beta|$. 
Proof. We have

$$
\sin \frac{\left|\theta-\theta_{\beta}\right|}{2}=\frac{h_{\theta}(\beta)}{|\beta|}=\frac{\mathrm{e}^{-t / 2} h_{\theta, t}(\beta)}{|\beta|} \leq \frac{\mathrm{e}^{-t / 2} \varepsilon^{\prime}}{|\beta|} .
$$

However since $h_{\theta,}(\beta) \leq \varepsilon^{\prime}$ and $t>0, h_{\theta}(\beta) \leq \varepsilon^{\prime} \leq|\beta| / 4$. Thus $v_{\theta}(\beta) \geq 3|\beta| / 4$ and we have

$$
\frac{3}{4}|\beta| \mathrm{e}^{-t / 2} \leq \mathrm{e}^{-t / 2} v_{\theta}(\beta) \leq \varepsilon^{\prime} \text { so } \mathrm{e}^{-t / 2} \leq \frac{4}{3} \frac{\varepsilon^{\prime}}{|\beta|}
$$

From (2.1) and (2.2),

$$
\sin \frac{\left|\theta-\theta_{\beta}\right|}{2} \leq \frac{4}{3} \frac{\left(\varepsilon^{\prime}\right)^{2}}{|\beta|^{2}} \leq \frac{1}{2}
$$

Thus

$$
\frac{\left|\theta-\theta_{\beta}\right|}{2} \leq 2 \sin \frac{\left|\theta-\theta_{\beta}\right|}{2}
$$

which together with (2.1) gives the result.

LEMMA 2.4. Further assume $\gamma$ is another saddle connection which crosses $\beta$ and $|\beta| \geq|\gamma|$. Further assume $\beta$ is $\varepsilon^{\prime}$-isolated with respect to $\varphi_{\theta, t}$. Then

$$
\left|\theta-\theta_{\gamma}\right| \geq \frac{C \varepsilon^{\prime} \mathrm{e}^{-t / 2}}{2|\gamma|} \text {. }
$$

Proof. Since $\gamma$ crosses $\beta$ and $\beta$ is $\varepsilon^{\prime}$-isolated, $|\gamma|_{\theta, t} \geq C \varepsilon^{\prime}$. Now

$$
v_{\theta, r}(\gamma) \leq \mathrm{e}^{-t / 2}|\gamma| \leq \mathrm{e}^{-t / 2}|\beta| \leq \frac{4}{3} \varepsilon^{\prime} \leq \frac{C}{2} \varepsilon^{\prime}
$$

by (2.2) and the definition of $C$. Therefore $h_{\theta, t}(\gamma) \geq \frac{1}{2} C \varepsilon^{\prime}$ which implies $h_{\theta}(\gamma) \geq$ $\frac{1}{2} C \mathrm{e}^{-t / 2} \varepsilon^{\prime}$ and therefore

$$
\frac{\left|\theta-\theta_{\gamma}\right|}{2} \geq \sin \frac{\left|\theta-\theta_{\gamma}\right|}{2}=\frac{h_{\theta}(\gamma)}{|\gamma|} \geq \frac{C}{2} \frac{\mathrm{e}^{-t / 2} \varepsilon^{\prime}}{|\gamma|}
$$

Proof of Proposition 2.1. Isolated curves with respect to a given metric can not cross and there are at most $C_{1}$ disjoint saddle connections. Thus we may assume all $\beta$ satisfy $|\beta| \geq 4 C_{2} \varepsilon$ and are $\varepsilon$-wide. Now suppose $\beta, \gamma \in S_{\sigma}(n)$, they cross and $|\beta| \geq|\gamma|$. Let $(\theta, t)$ be such that $|\beta| \leq \varepsilon$ and is isolated with respect to $\varphi_{\theta, t}$ and $v_{\theta, t}(\beta) \geq \varepsilon / C_{3}$. By Lemma 2.4 ,

$$
\frac{\left|\theta-\theta_{\gamma}\right|}{2} \geq \frac{C}{2} \frac{\mathrm{e}^{-t / 2} \varepsilon}{|\gamma|}
$$

Since Lemma 2.3 gives $\left|\theta-\theta_{\beta}\right| \leq 4 \mathrm{e}^{-t / 2} \varepsilon /|\beta|$ and $C>16$,

$$
\left|\theta_{\beta}-\theta_{\gamma}\right| \geq \frac{C}{4} \frac{\mathrm{e}^{-1 / 2}}{|\gamma|} \text {. }
$$

Now

$$
\frac{\varepsilon}{C_{3}} \leq \mathrm{e}^{-t / 2} v_{\theta}(\beta) \leq \mathrm{e}^{-t / 2}|\beta| \text { so } \mathrm{e}^{-t / 2} \geq \frac{\varepsilon}{|\beta| C_{3}}
$$

This gives

$$
\left|\theta_{\beta}-\theta_{\gamma}\right| \geq M /|\beta||\gamma|
$$


for $M$ depending only on $C, C_{3}, \varepsilon, \sigma$. Since both $\beta, \gamma$ have lengths in the interval $[\sigma n, n],\left|\theta_{\beta}-\theta_{\gamma}\right| \geq M^{\prime} / n^{2}$ for some $M^{\prime}$. Since there are at most $C_{1}$ saddle connections that do not cross, this inequality shows there are $0\left(n^{2}\right)$ saddle connections in $S_{\sigma}(n) \cap S^{\varepsilon}(n)$.

Proof of Proposition 2.2. Again we may assume $|\beta| \geq 4 C_{2} \varepsilon$. By rotation we can assume $\theta_{\alpha}=0$. Then $\beta D$-close to $\alpha$ means

$$
\sin \frac{\left|\theta_{\beta}\right|}{2}=\frac{h_{\theta_{\alpha}}(\beta)}{|\beta|} \leq \frac{D}{|\alpha||\beta|} .
$$

We first show there are 0 (1) $\beta$ such that $|\beta| \leq|\alpha|$, the bound independent of $|\alpha|$. Suppose two such $\beta_{i}$ cross and $\left|\beta_{1}\right| \geq\left|\beta_{2}\right|$. We may assume $\left|\theta_{\beta_{1}}-\theta_{\beta_{2}}\right| \leq \pi / 2$. Let $L$ be such that $|\alpha| \geq L\left|\beta_{1}\right|$. Then (2.4) gives

$$
\sin \frac{\left|\theta_{\beta_{i}}\right|}{2} \leq \frac{D}{L\left|\beta_{i}\right|\left|\beta_{1}\right|}, i=1,2
$$

By (2.3) we have

$$
\left|\theta_{\beta_{1}}-\theta_{\beta_{2}}\right| \geq \frac{M}{\left|\beta_{1}\right|\left|\beta_{2}\right|}
$$

since $\beta_{1}, \beta_{2}$ are $\varepsilon$-wide. Then for $L$ sufficiently large, depending only on $D$ and $M$ we have

$$
\left|\theta_{\beta_{1}}-\theta_{\beta_{2}}\right| \geq 4\left(\sin \frac{\left|\theta_{\beta_{1}}\right|}{2}+\sin \frac{\left|\theta_{\beta_{2}}\right|}{2}\right)
$$

which is impossible. Thus for some $L$ there are at most $C_{1}$ saddle connections $\beta$ with $|\beta| \leq|\alpha| / L$. Now for crossing $\beta_{i}$ with $|\alpha| \geq\left|\beta_{i}\right| \geq|\alpha| / L$, (2.3) shows $\left|\theta_{\beta_{1}}-\theta_{\beta_{2}}\right| \geq L^{\prime} /|\alpha|^{2}$ for some $L^{\prime}$. For $|\alpha|$ sufficiently large depending only on $D, L$ (2.4) shows $\theta_{\beta_{i}}$ is restricted to an interval of width $\left(2 D /|\alpha|^{2}\right) L$ about $\theta_{\alpha}=0$. Since $\left|\theta_{\beta_{1}}-\theta_{\beta_{2}}\right| \geq L^{\prime} /|\alpha|^{2}$, there can only be $0(1)$ such $\beta_{i}$. For $|\alpha|$ smaller than this fixed number, again $\left|\theta_{\beta_{1}}-\theta_{\beta_{2}}\right| \geq L^{\prime} /|\alpha|^{2}$ shows there are 0 (1) $\beta$.

Next consider crossing $\beta_{i}$ that satisfy $|\alpha| \leq \sigma n|\alpha| \leq\left|\beta_{i}\right| \leq n|\alpha|$. Now (2.3) gives $\left|\theta_{\beta_{1}}-\theta_{\beta_{2}}\right| \geq M / n^{2}|\alpha|^{2}$ for some $M$. If $\frac{1}{2} \sigma n|\alpha|^{2} \leq D$ there are only $0(n)$ such $\beta_{i}$. Thus we can assume $\frac{1}{2} \sigma n|\alpha|^{2} \geq D$. Then (2.4) gives

$$
\sin \frac{\left|\theta_{\beta_{i}}\right|}{2} \leq \frac{D}{|\alpha|\left|\beta_{i}\right|} \leq \frac{D}{|\alpha|^{2} \sigma n} \leq \frac{1}{2} \text { so } \frac{\left|\theta_{\beta_{i}}\right|}{2} \leq 2 \sin \frac{\left|\theta_{\beta_{i}}\right|}{2} \leq \frac{2 D}{|\alpha|^{2} \sigma n} .
$$

Thus the $\theta_{\beta_{i}}$ are restricted to an angle of width $0\left(1 / n|\alpha|^{2}\right)$ about 0 . Since they are $M / n^{2}|\alpha|^{2}$ apart there are at most $0(n)$ in the interval $[\sigma n, n]$. This proves the proposition.

Recall we need to consider disjoint systems of curves that simultaneously become short. We will need to know the number of such systems that contain a given saddle connection. In what follows $\Gamma=\left(\gamma_{1}, \ldots, \gamma_{p}\right)$ will be a system such that $\sigma^{i_{i}^{+1}} m \leq\left|\gamma_{i}\right| \leq \sigma^{\prime} \cdot m$ (respectively

$$
\left.\sigma^{l_{i}^{+}} m|\alpha| \leq\left|\gamma_{i}\right| \leq \sigma^{\prime} m|\alpha|\right)
$$


We will assume their lengths are in decreasing order with $0=l_{1} \leq l_{2} \leq \cdots \leq l_{p}$. We will also assume $t$ satisfies

$$
\frac{3}{4} \frac{\sigma n}{\varepsilon} \leq \mathrm{e}^{1 / 2} \leq \frac{2 n}{\varepsilon}
$$

(respectively $\frac{3}{4} \sigma n|\alpha| \leq \mathrm{e}^{t / 2} \leq 2 \varepsilon^{-1} n|\alpha|$ ) for some $n \geq m$. Next let

$$
U=U\left(m, l_{1}, \ldots, l_{p}\right)=\left\{\Gamma=\left(\gamma_{1}, \ldots, \gamma_{p}\right):\right.
$$

for some $(\theta, t), t$ in the given range, $\Gamma_{\theta, t}$ is isolated with respect to $\left.\varphi_{\theta, t}\right\}$ Let

$$
U_{i}=U_{i}\left(m, l_{i}, n\right)=\left\{\gamma_{i}: \gamma_{i} \text { is an element of a } p \text {-tuple in } U\left(m, l_{1}, \ldots, l_{p}, n\right) .\right\}
$$

Proposition 2.5. Card $U=O\left(\sigma^{-t_{i}}\right)$ card $U_{i}$ where the bound does not depend on $n$, $m, l_{i},|\alpha|$.

Proof. We prove this for $\sigma^{l_{i}+1} m \leq\left|\gamma_{i}\right| \leq \sigma^{i} i m$; the case with $|\alpha|$ is identical. Let $\gamma_{j}$ belong to a $p$-tuple with associated $(\theta, t)$. By (2.1) with $\varepsilon^{\prime}=\left|\gamma_{j}\right|_{\theta, t} \leq C_{2} \varepsilon$

$$
\sin \frac{\left|\theta-\theta_{\gamma_{j}}\right|}{2} \leq \frac{\varepsilon^{\prime} \mathrm{e}^{-t / 2}}{\left|\gamma_{j}\right|} \leq \frac{4}{3} \frac{\varepsilon}{\sigma n} \frac{\varepsilon^{\prime}}{\left|\Gamma_{j}\right|} \leq \frac{4}{3} \frac{\varepsilon^{2} C_{2}}{\sigma n} .
$$

If $\gamma_{j}^{\prime}$ crosses $\gamma_{j}$, then $\gamma_{j}$ isolated at time $t$ means $\mathrm{e}^{t / 2} h_{\theta}\left(\gamma_{j}^{\prime}\right) \geq \frac{1}{2} C \max \left(C \varepsilon, C \varepsilon^{\prime}\right)$ which implies

$$
\sin \left|\frac{\left(\theta-\theta_{y_{j}^{\prime}}\right)}{2}\right| \geq \frac{C}{2} \frac{\varepsilon}{2 n} \frac{\max \left(C \varepsilon, C \varepsilon^{\prime}\right)}{\left|\gamma_{j}^{\prime}\right|} .
$$

Now since $\gamma_{j}$ and $\gamma_{j}^{\prime}$ cross they cannot belong to the same $p$-tuple. Moreover if $\sigma^{l_{j}+1} m \leq\left|\gamma_{j}^{\prime}\right| \leq \sigma^{l} ; m$ as well, then 2.5, 2.6 and the fact that $C \geq 16 / \sigma$ means the angles $\theta$ about $\theta_{\gamma_{j}}$ and $\theta_{\gamma_{j}}$ are disjoint. In particular for some $M_{1}$ depending only on $C$, $\varepsilon, C_{2}$ and $\sigma$,

$$
\left|\theta_{\gamma_{j}}-\theta_{\gamma_{j}^{\prime}}\right| \geq M_{1} / n m \sigma^{t^{\prime}} \text {. }
$$

Now for $j>i, \sigma^{-t_{j}} \geq \sigma^{-t_{i}}$. Thus (2.5) and (2.7) mean that the $\theta_{\gamma_{j}}$ are further apart than the size of the interval of angles $\theta$ about $\theta_{\gamma_{i}}$. The fact that the intervals of $\theta$ about $\gamma_{j}$ and $\gamma_{j}^{\prime}$ are disjoint means for a given $\gamma_{i}$ there are $0(1) \gamma_{j}$ such that $\gamma_{i}, \gamma_{j}$ belong to the same $p$-tuple. For $j=i-1$ the lower bound (2.7) and the upper bound (2.5) on the size of the interval of $\theta$ about $\theta_{\gamma_{i}}$ show there are at most $0\left(\sigma^{l_{i-1}-l_{i}}\right) \gamma_{i-1}$ for each $\gamma_{i}$. Continuing, we find there are $0\left(\sigma^{t_{i-2}-t_{i-1}}\right) \gamma_{i-2}$ for each pair $\gamma_{i-1}, \gamma_{i}$; that is $0\left(\sigma^{i_{-2}-l_{i}}\right)$ triples. We continue in this manner and since $l_{1}=0$, there are $0\left(\sigma^{-l_{i}}\right)$ $p$-tuples $\left(\gamma_{1}, \ldots, \gamma_{p}\right)$ for each $\gamma_{i}$, proving the proposition.

To motivate the next proposition we recall the plan. For each $\beta$ which is not $\varepsilon$-wide we will associate a new Riemann surface $Y$ on which $\beta$ will have length $\varepsilon$. On $Y$ we will find a subcomplex with isolated boundary with $\beta$ in its interior. Consider this subcomplex simply as a topological surface with boundary without its metric structure. Other $\beta$ may determine the same topological surface considered as a subcomplex of a different Riemann surface with a different metric. We wish to consider one single image Riemann surface with a quadratic differential for the purposes of computing the total number of $\beta$ determining that topological surface (Theorem 4.1). A saddle connection $\beta$ which has length $\varepsilon$ with respect to one metric 
of course need not have length $\varepsilon$ with respect to another. The following computes the length of a curve with respect to an image metric when it is short with respect to another, provided both metrics make some other curve short.

LeMMA 2.6. Suppose $\beta_{1}, \beta_{2}$ are saddle connections that satisfy $4 C_{2} \varepsilon \leq \sigma n \leq\left|\beta_{i}\right| \leq n$ (respectively $\left.4 C_{2} \varepsilon \leq \sigma n|\alpha| \leq\left|\beta_{i}\right| \leq n|\alpha|\right)$ and $\left(\theta_{i}, t_{i}\right)$ satisfy $\left|\beta_{i}\right|_{\theta_{i}, t_{i}} \leq \varepsilon$ and $v_{\theta_{i}, t_{i}}\left(\beta_{i}\right) \geq$ $\varepsilon / C_{3}$. Suppose further $\gamma$ is disjoint from each, $|\gamma| \geq 4 C_{2} \varepsilon$ and $|\gamma|_{\theta_{1}, t_{i}}=\varepsilon_{i} \leq C_{2} \varepsilon$. Further suppose $\varepsilon_{2} \geq \varepsilon_{1}$. Then for some $M$ depending on $\varepsilon, \sigma$ but not on $\left|\beta_{i}\right|,|\gamma|$ or $\varepsilon_{i}$

$$
\frac{\left|\beta_{1}\right|_{\theta_{2}, t_{2}}}{|\gamma|_{\theta_{2}, \ell_{2}}} \leq \frac{M\left|\beta_{1}\right|}{|\gamma|} \text {. }
$$

Proof. Exactly as in Lemma 2.3

$$
\frac{1}{2}\left|\theta_{i}-\theta_{\gamma}\right| \leq \frac{2 \varepsilon_{i} \mathrm{e}^{-t_{i} / 2}}{|\gamma|} \leq \frac{2 \varepsilon_{2} \mathrm{e}^{-t_{i} / 2}}{|\gamma|} .
$$

By the conditions on $v_{\theta_{i}, i_{i}}\left(\beta_{i}\right)$ and the fact that the $\left|\beta_{i}\right|$ are bounded in terms of each other, the times $t_{1}, t_{2}$ are also. By (2.8)

$$
\sin \frac{\left|\theta_{1}-\theta_{2}\right|}{2} \leq \frac{\left|\theta_{1}-\theta_{2}\right|}{2} \leq \frac{M^{\prime} \varepsilon_{2} \mathrm{e}^{-t / 2}}{|\gamma|}
$$

for some $M^{\prime}$. Thus

$$
\frac{h_{\theta_{2}, t_{2}}\left(\beta_{1}\right)}{|\gamma|_{\theta_{2}, y_{2}}}=\frac{\mathrm{e}^{t_{2} / 2} h_{\theta_{2}}\left(\beta_{1}\right)}{\varepsilon_{2}}=\frac{\mathrm{e}^{t_{2} / 2}\left|\beta_{1}\right|}{\varepsilon_{2}} \sin \frac{\left|\theta_{2}-\theta_{2}\right|}{2} \leq \frac{M^{\prime}\left|\beta_{1}\right|}{|\gamma|} .
$$

On the other hand,

$$
\begin{aligned}
\frac{v_{\theta_{2}, t_{2}}\left(\beta_{1}\right)}{|\gamma|_{\theta_{2}, t_{2}}} & \leq \frac{2 \mathrm{e}^{-t_{2} / 2} v_{\theta_{2}}\left(\beta_{1}\right)}{\mathrm{e}^{-t_{2} / 2} v_{\theta_{2}}(\gamma)+\mathrm{e}^{t_{2} / 2} h_{\theta_{2}}(\gamma)} \\
& \leq \frac{2 v_{\theta_{2}}\left(\beta_{1}\right)}{v_{\theta_{2}}(\gamma)+\mathrm{e}^{t_{2} / 2} h_{\theta_{2}}(\gamma)} \leq \frac{2\left|\beta_{1}\right|}{|\gamma|} .
\end{aligned}
$$

Together this gives $\left|\beta_{1}\right|_{\theta_{2}, t_{2}} /|\gamma|_{\theta_{2}, t_{2}} \leq M\left|\beta_{1}\right| /|\gamma|$.

\section{Complexes with isolated boundaries}

The idea in this section is as follows. We start with $\beta$ on $X$ or on $Y$ and in that case disjoint from a saddle connection $\alpha$ on $Y$. We suppose $\beta$ is not $\varepsilon$-wide. We rotate so $\beta$ is vertical and contract the length to $\varepsilon$ using the Teichmüller map. By Proposition 1.11 we build an isolated separting system $\Gamma$ disjoint from $\beta$. We would like to count, using Propositions 2.1 (or Proposition 2.2) and 2.5, the number of $\Gamma$ that occur in this process. However the vertical length of $\Gamma$ may be $<\varepsilon / C_{3}$ at this time so $\Gamma$ may not be $\varepsilon$-wide. Thus Propositions 2.1 and 2.5 will not apply. If that is the case we go 'back' in time until the vertical length of $\Gamma$ is essentially $\varepsilon$ and then ask if it is still isolated. If so $\Gamma$ contains an $\varepsilon$-wide saddle connection $\gamma$ on $X$ (respectively $Y-\alpha$ ). We will be able to count the number of such $\gamma$ using Propositions 2.1 or 2.2 and then use Proposition 2.5 to count the $\Gamma$ that contain $\gamma$.

If $\Gamma$ is not isolated, again using Proposition 1.11 we build a further $\Gamma^{\prime}$ disjoint from $\Gamma$ and continue. By Proposition 1.11 this construction must end after $C_{1}$ steps 
with some $\varepsilon$-wide $\Gamma$. The following Proposition makes this precise. We are going to state it more generally with $\Gamma_{0}$, a system, instead of $\beta$ simply a saddle connection. The condition is there should be some $\theta, t$ such $|\Gamma|_{\theta, t}$ is short. This is clearly satisfied for $\beta$ a saddle connection with $\theta=\theta_{\beta}$. For systems that are not singletons there is also the added condition that at a later time the system is isolated. The added generality of considering systems is needed in the proof of the Theorem. Again $\mid$ without subscripts refers to lengths on the base surface $X$ (respectively $X-\alpha$ ). Also the angle $\theta$ will be suppressed as a subscript.

Proposition 3.1. Suppose $\Gamma_{0}$ is either a saddle connection or a system of disjoint saddles that separates the surface into two components. Suppose either $\left|\Gamma_{0}\right| \leq 4 C_{2} \varepsilon$ or for some $(\theta, t), t>0, \varepsilon / 2 \leq v_{\theta, t}\left(\Gamma_{0}\right) \leq\left|\Gamma_{0}\right|_{\theta, t} \leq \varepsilon$. Assume further in the second case, that if $\Gamma_{0}$ is not a singleton, there is a $s \geq t$ such that $\left|\Gamma_{0}\right|_{\theta, s} \leq \varepsilon$ and $\left(\Gamma_{0}\right)_{\theta, s}$ cannot be $\varepsilon$-extended. Then there is a number $M$ independent of $\left|\Gamma_{0}\right|$, a sequence $\Gamma_{0}, \Gamma_{1}, \ldots, \Gamma_{p}$, $p \leq C_{1}$, of mutually disjoint systems on $X$, (respectively $Y-\alpha$ ), a sequence of times $t_{1} \geq t_{2} \geq \cdots \geq t_{p} \geq t_{p+1} \geq 0$ such that

(0) If $\Gamma_{0}$ is $\varepsilon$-wide, $p=0, t_{1}=0$,

(i) $\left|\Gamma_{j}\right| \geq\left|\Gamma_{j+1}\right| / M$,

(ii) for each $j \geq 1, \Gamma_{j}$ divides $X$ (respectively $Y-\alpha$ ) into components $U_{j}$ and $V_{j}$ where $U_{j} \supsetneq U_{j+1}$ and $\Gamma_{0} \subset V_{1}$,

(iii) for $j \geq 1, \varepsilon^{\prime}=\left|\Gamma_{j}\right|_{t_{j}} \leq 4 C_{2}^{2} \varepsilon$ and $\Gamma_{j}$ is $\left(1, \varepsilon^{\prime}\right)$-isolated with respect to the metric defined by $\varphi_{i_{j}}$,

(iv) if $\left|\Gamma_{0}\right| \leq 4 C_{2} \varepsilon$ and is not $\varepsilon$-wide then $p=1, t_{1}=t_{2}=0 ; \varepsilon^{\prime} \leq\left|\Gamma_{1}\right| \leq 4 C_{2}^{2} \varepsilon$ and $\Gamma_{1}$ is $\left(1, \varepsilon^{\prime}\right)$-isolated,

(v) if neither (0) or (iv) holds, then $t_{1}=t>0$ and for $1 \leq j<p, v_{t_{i}}\left(\Gamma_{j}\right)<\varepsilon / C_{3}$ and $t_{j+1}<t_{j}$.

(a) If in addition $\left|\Gamma_{j}\right| \leq 4 C_{2} \varepsilon$ then $p=j+1, t_{p+1}=t_{p}=0$ and $\varepsilon^{\prime}=\left|\Gamma_{p}\right| \leq 4 C_{2}^{2} \varepsilon$ and $\Gamma_{p}$ is $\left(1, \varepsilon^{\prime}\right)$-isolated.

(b) If on the other hand $\left|\Gamma_{j}\right|>4 C_{2} \varepsilon$, then $\varepsilon / 2 \leq v_{t_{j+1}}\left(\Gamma_{j}\right) \leq\left|\Gamma_{j}\right|_{t_{j+1}} \leq \varepsilon$ so $t_{j+1}>0$.

(vi) If $(\mathrm{v})(\mathrm{a})$ does not hold then either $v_{t_{p}}\left(\Gamma_{p}\right) \geq \varepsilon / C_{3}$ and $t_{p+1}=t_{p}$ or $v_{t_{p}}\left(\Gamma_{p}\right)<\varepsilon / C_{3}$. In that case either $\left|\Gamma_{p}\right| \leq 4 C_{2} \varepsilon$ and $t_{p+1}=0$ or $\left|\Gamma_{p}\right|>4 C_{2} \varepsilon$ and $\varepsilon / 2 \leq v_{t_{n+1}}\left(\Gamma_{p}\right) \leq\left|\Gamma_{p}\right|_{t_{p+1}} \leq \varepsilon$. In all cases $\Gamma_{p}$ is $\varepsilon$-wide.

(vii) For each $0 \leq j \leq p-1$ there is a saddle connection $\gamma_{j} \in \Gamma_{j}-\Gamma_{j+1}$ such that

(a) $\left|\gamma_{j}\right|_{t_{j+1}} \geq\left|\Gamma_{j+1}-\Gamma_{j}\right| / C_{4}$

(b) there is no $\Gamma C_{5}=8 C_{4}$-separating $\gamma_{j}$ from $\Gamma_{j+1}$ at time $t_{j+1}$.

(c) $v t_{j+1}\left(\gamma_{j}\right) \geq \frac{1}{2} h_{t_{j+1}}\left(\gamma_{j}\right)$ if $t_{j+1}>0$.

Remarks. We will use either (iv), (v)(a), or (vi) and Propositions 2.1 or 2.2, and Proposition 2.5 to calculate the number of $\Gamma_{p}$ that occur. (vii) is designed so that with Theorem 4.1 for each $\Gamma_{j+1}$ we can compute the number of $\gamma_{j}$ and therefore by Proposition 2.5 and (iii) the number of $\Gamma_{j}$ that occur. In particular (vii)(c) is necessary to control the length of $\gamma_{j}$ on $X$.

Proof. To simplify notation let $\bar{\varepsilon}=4 C_{2} \varepsilon$. $\Gamma_{0}$ has a complementary component $U$ with area $\geq \frac{1}{2}$. If $\Gamma_{0}$ is already $\varepsilon$-wide there is no construction; $(0)$ holds. If $\left|\Gamma_{0}\right| \leq \bar{\varepsilon}$ and is not $\varepsilon$-wide form the system $\Gamma_{1}$ and complementary component $U_{1}$ provided 
by Proposition 1.11. Area $U_{1} \geq g\left(\frac{1}{2}\right)$. We set $p=1, t_{1}=t_{2}=0$ in this case. Now (i)-(iv) are automatically satisfied by construction. (vii)(a) and (b) follow from Corollary 1.12. We are done in this case. If $\left|\Gamma_{0}\right|>\bar{\varepsilon}$ let $t=t_{1}$. On the surface $X_{t_{1}}$ (respectively $\left.Y_{t_{1}}-\alpha\right)$ we have $\varepsilon / 2 \leq v_{t_{1}}\left(\Gamma_{0}\right) \leq\left|\Gamma_{0}\right|_{t_{1}} \leq \varepsilon$ and $\Gamma_{0}$ not isolated. Let $\left(\Gamma_{1}, U_{1}\right)$ denote the system with complementary component given by Proposition 1.11; Area $U_{1} \geq g\left(\frac{1}{2}\right)$ and $\left|\Gamma_{1}\right|_{t_{1}} \leq C_{2} \varepsilon$. If $v_{t_{1}}\left(\Gamma_{1}\right) \geq \varepsilon / C_{3}$ we set $p=1 ; t_{1}=t_{2}$ and the construction ends. Now we have to check (ii) and (vii). Here (i), (iii), (vi) are satisfied by definition and (iv) and (v) are vacuous. To see (ii), the fact that $\left|\Gamma_{0}\right|_{t_{1}} \geq M\left|\Gamma_{1}\right| t_{1}$ for some $M$ and the fact that at time $t_{1}$ the ratio of vertical to horizontal length of $\Gamma_{0}$ is bounded away from zero means the inequality between $\left|\Gamma_{0}\right|_{t_{1}}$ and $\left|\Gamma_{1}\right|_{t_{1}}$ persists up to another universal factor at an earlier time $t=0$.

Checking (vii)(c) is more complicated if $\Gamma_{0}$ is not a singleton saddle connection. The point is the $\gamma_{0}^{\prime}$ provided by Corollary 1.12 is not necessarily the saddle connection $\gamma_{0}$ that satisfies $\varepsilon / 2 \leq v_{t_{1}}\left(\gamma_{0}\right) \leq\left|\Gamma_{0}\right|_{t_{1}} \leq \varepsilon$. We argue therefore as follows. If there is no $\Gamma C_{5}$-separating $\gamma_{0}$ from $\Gamma_{1}$, then $\gamma_{0}$ satisfies (vii). If there is such a $\Gamma$ then $\left|\Gamma_{1}\right|_{t_{1}} \geq C_{5}|\Gamma|_{t_{1}}$. Then $\left|\gamma_{0}^{\prime}\right|_{t_{1}} \geq\left|\Gamma_{1}\right|_{t_{1}} / C_{4} \geq\left(C_{5} / C_{4}\right)|\Gamma|_{t_{1}}$. At the later time $s \geq t_{1}$ we claim $|\Gamma|_{s} \geq\left|\Gamma_{0}\right|_{s}$. To prove the claim assume otherwise and consider the combination $\Gamma^{\prime}$ of $\Gamma$ and $\Gamma_{0}$ at time $s$. Then

$$
\left|\Gamma^{\prime}\right|_{s} \leq C\left(\left|\Gamma_{0}\right|_{s}+|\Gamma|_{s}\right) \leq 2 C_{1}\left|\Gamma_{0}\right|_{s} \leq(C+1)\left|\Gamma_{0}\right|_{s},
$$

the last inequality by the definition of $C$. Also $\Gamma^{\prime}$ separates $\Gamma_{0}$ from $\Gamma_{1}$. Now we consider the region $X^{\prime}$ bounded by $\Gamma^{\prime}$ and $\Gamma_{0}$ inside the complex $X_{1}$ bounded by $\Gamma_{0}$ and $\Gamma_{1}$. At time $t_{1}, X_{1}$ has area $\leq A\left(C_{2} \varepsilon\right)$ and since area is preserved under the Teichmüller flow, this is true at time $s$ as well. Therefore $X_{2}$ has area $\leq A\left(C_{2} \varepsilon\right)$ and since

$$
\left|\Gamma^{\prime}\right|_{s} \leq(C+1)\left|\Gamma_{0}\right|_{s} \leq(C+1) \varepsilon,
$$

$X_{2}$ is a $C_{2} \varepsilon$-extension of $\Gamma_{0}$ at time $s$, a contradiction to the assumption proving the claim. Thus as time goes from $t_{1}$ to $s,\left|\gamma_{0}^{\prime}\right|$ decreases from $\geq 8|\Gamma|_{t_{1}}$ to a number $\leq\left|\Gamma_{0}\right|_{s} \leq|\Gamma|_{s}$. If the ratio in length of two saddle connections goes from at least eight to less than one in positive time, the first cannot be nearly horizontal. A definite estimate gives (vii)(c). We have found $\gamma_{0}^{\prime}$ and we are done in this case.

If $v_{t_{1}}\left(\Gamma_{1}\right)<\varepsilon / C_{3}$ and $\left|\Gamma_{1}\right| \leq \bar{\varepsilon}$ set $t_{2}=0$. If $\Gamma_{1}$ is isolated at $t_{2}=0$ (on $X$ or $Y-\alpha$ ) set $p=1$. Again the construction ends and (i)-(vii) are satisfied just as in the last case. If $\Gamma_{1}$ is not isolated we find $\Gamma_{2}$ given by Proposition 1.11. The existence is guaranteed by the fact that area $U_{1} \geq \delta_{1}=g\left(\frac{1}{2}\right)$. Then we set $p=2$ and $t_{3}=0$. Now (vii)(c) is satisfied at $t_{2}=0$ because just as in the argument above there is a later time, namely $t_{1}$, where $\Gamma_{1}$ cannot be $C_{2} \varepsilon$-extended.

What remains is the case $v_{t_{1}}\left(\Gamma_{1}\right)<\varepsilon / C_{3}$ and $\left|\Gamma_{1}\right| \geq \bar{\varepsilon}$. Choose $\gamma_{1}$ so $v_{t_{1}}\left(\gamma_{1}\right)=v_{t_{1}}\left(\Gamma_{1}\right)$. Find $t_{2}<t_{1}$ so $v_{t_{2}}\left(\gamma_{1}\right)=\varepsilon / \sqrt{2}$. That is, go back in time, increasing the vertical length of $\gamma_{1}$ until it is $\varepsilon / \sqrt{2}$. Now

$$
v_{t_{1}}\left(\gamma_{1}\right)<\varepsilon / C_{3} \text { implies } \mathrm{e}^{\left(t_{1}-t_{2}\right) / 2} \geq C_{3} / \sqrt{2} \text {. }
$$

Also $\left|\Gamma_{1}\right| \geq \bar{\varepsilon}$ and $h_{t_{1}}\left(\Gamma_{1}\right) \leq C_{2} \varepsilon$ imply $t_{2} \geq 0$. For any $\gamma \in \Gamma_{1}, h_{t_{1}}(\gamma) \leq C_{2} \varepsilon$ and 
$\mathrm{e}^{\left(t_{1}-t_{2}\right) / 2} \geq C_{3} / \sqrt{2}$ imply $h_{t_{2}}(\gamma)<\varepsilon \sqrt{2} / 4$ and therefore

$$
|\gamma|_{t_{2}} \leq\left(\left(\frac{\varepsilon \sqrt{2}}{4}\right)^{2}+\left(\frac{\varepsilon}{\sqrt{2}}\right)^{2}\right)^{1 / 2} \leq \varepsilon .
$$

If $\Gamma_{1}$ is isolated at $t=t_{2}$, we set $p=1$, the construction ends; (i)-(vii) are satisfied as before. If not, construct $\left(\Gamma_{2}, U_{2}\right)$ by Proposition 1.11. $U_{2} \subset U_{1}$, area $U_{2} \geq \delta_{2}=$ $g\left(\delta_{1}\right)$. Once again (i)-(vii) are satisfied by the same arguments. We continue this process. After $p \leq C_{1}$ steps it must stop. Since $\delta_{C_{1}}>0$ it must end with some $\left(\Gamma_{p}, U_{p}\right)$ for which there is no $\Gamma_{p+1}$. This means either $v_{t_{p}}\left(\Gamma_{p}\right) \geq \varepsilon / C_{3}$ and it is isolated, or if not, either $\left|\Gamma_{p}\right| \leq 4 C_{2} \varepsilon$ and $\Gamma_{p}$ is isolated at $t_{p+1}=0$ or for some $t_{p+1}<t_{p}, \varepsilon / 2 \leq$ $v_{t_{p+1}}\left(\Gamma_{p}\right) \leq|\Gamma|_{p+1} \leq \varepsilon$ and $\Gamma_{p}$ is isolated there. For if it were not isolated at these three possibilities we could build a further $\Gamma_{p+1}$.

Suppose now the following situation occurs as in the conclusion to Proposition 3.1. There is a saddle connection $\tau$, a pair $(\theta, t)=(\theta(\tau), t(\tau))$ and constants $M_{1}$, $M_{2}$ independent of $\tau$ such that

(i) $|\tau|_{\theta, t} \leq \varepsilon^{\prime} \leq \varepsilon, v_{t}(\tau) \geq \varepsilon^{\prime} / C_{3}$.

(ii) there is a system $\Gamma$ disjoint from $\tau$ such that $|\Gamma|_{\theta, r} \leq M_{1}|\tau|_{\theta, r}$.

(iii) $\tau$ is not $M_{2}$-separated from $\Gamma$ at time $t$.

Under these circumstances we will say $\Gamma$ is $\varepsilon^{\prime}$-associated to $\tau$. Let $B_{L}(\Gamma)=\{\tau: \Gamma$ is $\varepsilon$-associated to $\tau, \sigma L \leq|\tau| \leq L$, and $\left.|\Gamma| \geq 4 C_{2} \varepsilon\right\}$. Let $\varepsilon_{0}=\max _{\tau \in B_{L}(\Gamma)}|\Gamma|_{\theta(\tau), r(\tau)}$, the maximum taken on for some $\tau_{0}$ and $\left(\theta_{0}, t_{0}\right)=\left(\theta_{0}\left(\tau_{0}\right), t_{0}\left(\tau_{0}\right)\right)$. By Lemma 2.6, for any $\tau \in B_{L}(\Gamma)$,

$$
\frac{|\tau|_{\theta_{0}, t_{0}}}{\varepsilon_{0}} \text { is } 0\left(\frac{|\tau|}{|\Gamma|}\right)=0\left(\frac{L}{|\Gamma|}\right) .
$$

By assumption $\tau$ is not $M_{2}$-separated from $\Gamma$ on the surface $Y_{\theta(\tau), t(\tau)}$ but it may be so separated on $Y_{\theta_{0}, l_{0}}$. We will wish to compute card $B_{L}(\Gamma)$ in Theorem 4.1 making the computations on the surface $Y_{\theta_{0}, t_{0}}$ where the separating hypothesis is necessary for that theorem.

The purpos: of the following lemma is to show there are a bounded number of $\tau_{i} \in B_{L}(\Gamma)$ and a number $M_{3}$ such that for each $\tau$ there is a $\tau_{i}$ such that $\tau$ is not $M_{3}$-separated on $Y_{\theta\left(\tau_{i}\right), t\left(\tau_{i}\right)}$.

LEMMA 3.2. There are sets $B_{i} \subset B(\Gamma), i \leq C_{1}$, and $M_{3}=M_{3}\left(M_{1}, M_{2}\right)$ such that

(i) $B_{L}(\Gamma)=U_{i} B_{i}$,

(ii) for each $i$ there is a $\tau_{i} \in B_{i}$ and $\left(\theta_{i}, t_{i}\right)=\left(\theta_{i}\left(\tau_{i}\right), t\left(\tau_{i}\right)\right)$ such that for all $\tau \in B_{i}$,

$$
\frac{|\tau|_{\theta_{i}, t_{i}}}{|\Gamma|_{\theta_{i}, t_{i}}} \text { is } 0\left(\frac{L}{|\Gamma|}\right)
$$

(iii) $\tau \in B_{i}$ is not $M_{3}$-separated from $\Gamma$ on $Y_{i}=Y_{\theta_{i}, t_{i}}$.

Proof. Let $B_{0}=\left\{\tau \in B_{L}(\Gamma): \tau\right.$ is not $\left(2 C_{1}\right)^{C_{1}} M_{2}$-separated from $\Gamma$ on $\left.Y_{0}=Y_{\theta_{0}, t_{0}}\right\}$. Suppose $B_{0} \neq B_{L}(\Gamma)$. By taking at most $C_{1}$ combinations of various $\Gamma^{\prime}$ that do $\left(2 C_{1}\right)^{C_{1}} M_{2}$-separate various $\tau \in B_{L}(\Gamma)$ from $\Gamma$ and using Corollary 1.4 we find a $\Gamma^{\prime}$ that $M_{2}$-separates every $\tau \in B_{L}(\Gamma)-B_{0}$ from $\Gamma$ on $Y_{0}$. Let $U^{\prime}$ the component of $Y_{0}-\Gamma^{\prime}$ containing such $\tau$ and $V^{\prime}$ the complementary component. Now consider 
$\max _{r \in B_{L}(\Gamma)-B_{0}}|\Gamma|_{\theta, r}$. Suppose the maximum is taken on by $\tau_{1}$ at $\left(\theta_{1}, t_{1}\right)$ with corresponding $Y_{1}$. By assumption $\left|\Gamma^{\prime}\right|_{\theta_{1}, t_{1}} \geq|\Gamma|_{\theta_{1}, t_{1}} / M_{2}$ since $\tau_{1}$ is not $M_{2}$-separated from $\Gamma$ on $Y_{1}$. Now let $B_{1}=\left\{\tau \in B_{L}(\Gamma)-B_{0}: \tau\right.$ is not $\left(2 C_{1}\right)^{C_{1}} M_{2}$-separated from $\Gamma$ on $\left.Y_{1}\right\}$. Again (ii) is satisfied for $\tau \in B_{1}$ by Lemma 2.6. Again it is possible $B_{L}(\Gamma)-B_{0}-B_{1} \neq \varnothing$. That is, there is a $\tau \notin B_{0} \cup B_{1}$ which is $\left(2 C_{1}\right)^{C_{1}} M_{2}$-separated by $\Gamma^{\prime \prime}$ from $\Gamma$ on $Y_{1}$. Such a $\Gamma^{\prime \prime}$ must intersect $U^{\prime}$ for otherwise $\tau_{1}$ itself would be so separated. As before taking at most $C_{1}$ combinations of such $\Gamma^{\prime \prime}$ and then the combination of the resulting system with $\Gamma^{\prime}$ we find $\Gamma^{\prime \prime}$ separating all $\tau \epsilon$ $B_{L}(\Gamma)-B_{1}-B_{2}$ from $\Gamma$. Now $\Gamma^{\prime \prime} \subset U^{\prime}$. Let $U^{\prime \prime}$ be the complementary component containing all such $\tau$.

We repeat the maximizing procedure to find a new $\tau_{2}$ and a new $B_{2}$. Since the $U^{\prime \prime}$ are decreasing, the process ends after at most $C_{1}$ steps and

$$
B_{L}(\Gamma)=\bigcup_{i=0}^{p} B_{i}
$$

\section{Proof of the theorems}

We collect our results in this section and prove both the preliminary Theorem 4.1 and the main theorem.

THEOREM 4.1. There exists $k=k\left(\varepsilon, M, C_{1}\right)$ such that card $T(m, \alpha, M)$ is $0\left(m(\log m)^{k}\right)$.

We will adopt the following terminology. Suppose $\Omega, \Gamma$ are disjoint systems; by card $\{\Omega \mid \Gamma\}$ will refer to the number of $\Omega$ disjoint from $\Gamma$ for a given $\Gamma$.

Proof of Theorem 4.1. We start by remarking that $|\beta| \geq|\alpha| / M$ since $\beta$ is not $M$-separated from $\alpha$.

The proof is by induction on the number, $r \leq C_{1}$, of disjoint segments that can be added to $Y-\alpha$. At each stage of the induction the exponent $k$ can increase by a fixed amount. Since the induction is of length $\leq C_{1}$ the final exponent will still be bounded. At each stage we will denote this exponent as $k$ even as it changes.

If $r=1$ then either $Y$ is simply connected or is an annulus. If $Y$ is simply connected it is either a quadrilateral and $\alpha$ is on the boundary or has five sides and $\alpha$ crosses the domain. In either case card $T(m, \alpha)=2$.

If $Y$ is an annulus, $\alpha$ crosses from one boundary to the other. Then $\alpha$, an edge of the boundary of $Y$, and $\beta$ bound a triangle so $\beta$ and $\alpha$ are 2-close. If $|\beta| \leq 4 C_{2} \epsilon$ it is isolated in $Y-\alpha$ since there can be no $\varepsilon$ complex disjoint from $\alpha$ containing $\beta$. Similarly if $|\beta|>4 C_{2} \varepsilon$ it is $\varepsilon$-wide. Thus card $T(m, \alpha)=\operatorname{card} T^{\varepsilon}(m, \alpha, 2)=0(m)$ by Proposition 2.2 .

Now suppose the theorem is true whenever fewer than $r_{0}$ curves can be added and $r_{0}$ trajectories can be added to $Y-\alpha$. This is the induction hypothesis in place for the rest of the proof. There are several cases to consider.

Case $I$. $|\beta| \leq 4 C_{2} \varepsilon$. Form the complex containing $\beta$ with isolated boundary $\Gamma_{1}$ with longest curve $\gamma_{1}$. There are $O(1)$ such $\Gamma_{1}$ since it is isolated on $Y$. If $\Gamma_{1}$ does not separate $\beta$ from $\alpha$ then $\beta$ and $\alpha$ are contained in a smaller complex $Y-\Gamma_{1}$ to which 
fewer than $r_{0}$ curves can be added. For each such $\Gamma_{1}$ apply the induction hypothesis. If $\Gamma_{1}$ does separate, then by assumption $\left|\gamma_{1}\right|=\left|\Gamma_{1}\right| \geq|\alpha| / M$. Consider the complex $Z$ containing $\beta$ bounded by $\Gamma_{1}$ where the quadratic differential is renormalized so that $Z$ has unit area. Fewer than $r_{0}$ trajectories can be added. We apply the induction hypothesis to $\beta$ and $\gamma_{1}$. Since $\left|\gamma_{1}\right| /|\alpha| \geq M^{-1}$ and there are $0(1)$ such $\Gamma_{1}$ we are done. There are $0\left(m(\log m)^{k}\right)$ such $\beta$.

Case II. $|\beta| \geq 4 C_{2} \varepsilon$. Consider the sequence $\Gamma_{1}, \ldots, \Gamma_{p}$ and times $t_{1}, \ldots, t_{p+1}$ constructed in Proposition 3.1.

Recall either $\Gamma_{p}$ is $\varepsilon$-wide or isolated on $Y-\alpha$. Recall also $\left|\Gamma_{i}\right|_{t_{i+1}} \geq\left|\Gamma_{i+1}\right|_{t_{i+1}} / M$ for some constant $M$. The first possibility is $\left|\Gamma_{p}\right|<4 C_{2} \varepsilon$. The cardinality of such $\Gamma_{p}$ is 0 (1) since $\Gamma_{p}$ is isolated. Then whether or not $\Gamma_{p}$ separates $\beta$ from $\alpha$ we argue exactly as in the previous two paragraphs when considering $|\beta| \leq 4 C_{2} \varepsilon$. Thus we may assume $\left|\Gamma_{p}\right| \geq 4 C_{2} \varepsilon$.

We now make the additional assumption, to be removed later, that $\beta$ is $D$-close to $\alpha$. Then by Lemma 1.2 and induction each $\Gamma_{i}$ is $D^{\prime}$-close to $\alpha$ for $D^{\prime}$ depending on $D$.

If $\left|\Gamma_{p}\right| \leq|\alpha|$ then by Proposition 2.2 and Proposition 2.5 there are $0(1)$ such $\Gamma_{p}$. Again whether or not $\Gamma_{p}$ divides we apply the induction hypothesis to $\beta$ and $Y-\Gamma_{p}$. Again note if $\Gamma_{p}$ does divide, $\left|\Gamma_{p}\right| \geq|\alpha| / M$. Thus we may assume $\left|\Gamma_{p}\right| \geq|\alpha|$. Now let $V(m, \alpha)$ be the set of systems $\Gamma$ such that

(a) $\Gamma$ is either a saddle connection or divides $Y-\alpha$ into two components,

(b) $|\Gamma| \leq m|\alpha|$,

(c) $\Gamma$ is $D^{\prime}$-close to $\alpha$, a universal $D^{\prime}$,

(d) if $|\Gamma| \geq 4 C_{2}^{2} \varepsilon$ there is some $t>0$ such that $\varepsilon / 2 \leq v_{t}(\Gamma) \leq|\Gamma|_{1} \leq \varepsilon$ and if $\Gamma$ is not a singleton, some $t^{\prime} \geq t$ such that $|\Gamma|_{t^{\prime}} \leq C_{2}^{2} \varepsilon$ and $\Gamma_{t^{\prime}}$ cannot be $C_{2} \varepsilon$-extended. if $4 C_{2} \varepsilon \leq|\Gamma| \leq 4 C_{2} \varepsilon$ then either $\Gamma$ is isolated or there is some $t$ as above, and if $\Gamma$ is not a singleton, some $t^{\prime}$ as above.

if $|\Gamma| \leq 4 C_{2} \varepsilon$ and $\Gamma$ is not a singleton, there is some $t^{\prime} \geq 0$ as above.

(e) for each $\Gamma$ determining a sequence $\Gamma=\Gamma_{1}, \ldots, \Gamma_{p}$ by Proposition 1.11, $\left|\Gamma_{p}\right| \geq|\alpha|$.

Claim. card $V(m, \alpha)=0\left(m(\log m)^{k}\right)$.

To prove the claim, for any $\Gamma_{0} \in V(m, \alpha)$ consider the sequence $\Gamma_{0}, \ldots, \Gamma_{p}$ constructed in Proposition 3.1. The proof is by induction on the length of the sequence. Let $\gamma_{i}$ be the longest curve on $\Gamma_{i}$. If $p=0, \Gamma_{0}$ is already isolated at time $t$. Then card $\left\{\gamma_{0}\right\}$ is $0(m)$ by Proposition 2.1 and Card $\left\{\Gamma_{0}\right\}$ is $0(m)$ by Proposition 2.5.

The induction hypothesis is card $\left\{\Gamma_{0} \in V(m)\right\}$ is $0\left(m(\log m)^{k}\right)$ for the set of $\Gamma_{0}$ that determine sequences $\Gamma_{0}, \ldots, \Gamma_{j}, j \leq p-1$ of length $\leq p$. Now suppose $\Gamma_{0}$ determines a sequence $\Gamma_{0}, \ldots, \Gamma_{p}$ of length $p+1$. Then $\sigma^{t_{1}+1} m|\alpha| \leq\left|\Gamma_{1}\right| \leq \sigma^{\prime} m|\alpha|$ where $M_{1} \leq l_{1} \leq M_{2} \log m_{1}$ the last inequality by the assumption on $\left|\Gamma_{p}\right|$ and Proposition 3.1(i).

Now $\Gamma_{1} \in V\left(\sigma^{\prime} i m, \alpha\right)$ by Proposition 3.1. Now $\Gamma_{1}$ determines a sequence $\Gamma_{1}, \ldots, \Gamma_{p}$ of length $p$. By the induction hypothesis

$$
\operatorname{card}\left\{\Gamma_{1}\right\}=0\left(\sigma^{l} \cdot m\left(\log \left(\sigma^{\prime} \cdot m\right)\right)^{k}\right) .
$$


By Proposition 3.1(vii) there is a $\gamma_{0}^{\prime} \in \Gamma_{0}$ such that $\gamma_{0}^{\prime}$ is not $C_{5}$-separated from $\Gamma_{1}$ at time $t_{1}$. If $t_{1}>0, v_{t_{1}}\left(\gamma_{0}^{\prime}\right) \geq \frac{1}{2} h_{t_{1}}\left(\gamma_{0}^{\prime}\right)$ as well. This implies for $t_{1}>0$

$$
\begin{aligned}
\left|\gamma_{0}^{\prime}\right| & \geq \mathrm{e}^{t_{1} / 2} v_{t_{1}}\left(\gamma_{0}^{\prime}\right) \geq M \mathrm{e}^{t_{1} / 2}\left|\gamma_{0}^{\prime}\right|_{t_{1}} \geq M \mathrm{e}^{t_{1} / 2}\left|\Gamma_{1}\right|_{t_{1}} / C_{5} \\
& \geq M \mathrm{e}^{t_{1} / 2}\left(h_{t_{1}}\left(\Gamma_{1}\right)+v_{t_{1}}\left(\Gamma_{1}\right)\right) \geq M\left|\Gamma_{1}\right|
\end{aligned}
$$

for a set of different constants $M$. If $t_{1}=0,\left|\gamma_{0}^{\prime}\right| \geq M\left|\Gamma_{1}\right|$ by Proposition 1.11 directly. Thus $\sigma^{j_{0}+1} m|\alpha| \leq\left|\gamma_{0}^{\prime}\right| \leq \sigma^{j_{0}} m|\alpha|$ where $M_{4} \leq j_{0} \leq M_{3} l_{1}$. Moreover if $t_{1}>0, v_{t_{1}}\left(\gamma_{0}^{\prime}\right) \geq$ $\frac{1}{2} h_{t_{2}}\left(\gamma_{0}^{\prime}\right)$ implies $v_{t_{1}}\left(\gamma_{0}^{\prime}\right) \geq\left|\gamma_{0}^{\prime}\right| t_{1} / C_{3}$. We are now in a position to apply first Lemma 3.2 and then the induction hypothesis on the number of curves.

First by Lemma 3.2 there are $s \leq C_{1}$ pairs $\left(\theta_{i}, t_{i}\right)$ such that for each such $\gamma_{0}^{\prime}$ there is a $\left(\theta_{i}, t_{i}\right)$ such that

$$
\frac{\left|\gamma_{0}^{\prime}\right|_{\theta_{i,} t_{i}}}{\left|\Gamma_{1}\right|_{\theta_{i}, t_{i}}} \text { is } 0\left(\frac{\left|\gamma_{0}^{\prime}\right|}{\left|\Gamma_{1}\right|}\right)=0\left(\sigma^{j_{0}-l_{1}}\right)
$$

and $\gamma_{0}^{\prime}$ is not $M_{7}$-separated from $\Gamma_{1}$ on $Y_{\theta_{i}, t_{i}}$. Renormalize each such $Y_{\theta_{i}, t_{j}}$ to have unit area. Fewer than $r_{0}$ saddle connections can be added to $Y_{\theta_{i}, i_{i}}$. By the induction hypothesis on the number of curves,

$$
\operatorname{card}\left\{\gamma_{0}^{\prime} \mid \Gamma_{1}\right\} \text { is } 0\left(\frac{\left|\gamma_{0}^{\prime}\right|_{\theta_{i,}, t_{i}}}{\left|\Gamma_{1}\right|_{\theta_{i}, t_{i}}}\left(\log \frac{\left|\gamma_{0}^{\prime}\right|_{\theta_{i, t_{i}}}}{\left|\Gamma_{1}\right|_{\theta_{i,}, t_{i}}}\right)^{k}\right)=0\left(\sigma^{j_{0}-l_{1}}\left(l_{1}-j_{0}\right)^{k}\right) \text {. }
$$

Now by Proposition 2.5,

$$
\operatorname{card}\left\{\Gamma_{0} \mid \Gamma_{1}\right\}=0\left(\sigma^{-j_{0}}\right) 0\left(\sigma^{j_{0}-l_{1}}\right)\left(l_{1}\right)^{k}=0\left(\sigma^{-l_{1}} l_{1}^{k}\right) .
$$

To find card $\left\{\Gamma_{0}\right\}$ we multiply this quantity by card $\left\{\Gamma_{1}\right\}$ and sum over the possible lengths for $\Gamma_{1}$ and $\gamma_{0}^{\prime}$. That is,

$$
\begin{aligned}
\operatorname{card}\left\{\Gamma_{0}\right\} & =\sum_{l_{1}=M_{1}}^{M_{2} \log m} \sum_{j_{0}=M_{3}}^{M_{4} \log l_{1}} 0\left(\sigma^{-l_{1}} l_{1}^{k}\right) \sigma^{l_{1}} m\left(\log \sigma^{l_{1}} m\right)^{k} \\
& =0\left(m(\log m)^{k}\right)(\text { different } k) .
\end{aligned}
$$

This proves the claim.

To calculate $\{\beta\}$ we proceed in much the same way. For each $\beta$ consider the sequence $\beta=\Gamma_{0}, \ldots, \Gamma_{p}$ determined by Proposition 3.1. We have $\sigma^{l_{1}+1} m|\alpha| \leq\left|\Gamma_{1}\right| \leq \sigma^{\prime} m|\alpha|$ where $m_{1} \leq l_{1} \leq M_{2} \log m$ and $\Gamma_{1} \in V\left(\sigma^{\prime} m, \alpha\right)$. By the claim

$$
\operatorname{card}\left\{\Gamma_{1}\right\}=0\left(\sigma^{l_{1}} m\left(\log \sigma^{\prime} m\right)^{k}\right) \text {. }
$$

To calculate card $\left\{\beta \mid \Gamma_{1}\right\}$ we proceed in exactly the same way. On the surface bounded by $\Gamma_{1}$ containing $\beta$ renormalize so the area is 1. By Lemma 3.2 and Proposition 3.1 there are $s \leq C_{1}$ pairs $\left(\theta_{i}, t_{i}\right)$ such that for each $\beta$ there is an $\left(\theta_{i}, t_{i}\right)$ such that

$$
\frac{|\beta|_{\theta_{i}, t_{i}}}{\left|\Gamma_{1}\right|_{\theta_{i}, t_{i}}} \text { is } 0\left(\frac{|\beta|}{\left|\Gamma_{1}\right|}\right)=0\left(\sigma^{-t_{1}}\right)
$$

and $\beta$ is not $M_{6}$-separated from $\Gamma_{1}$ with respect to $\varphi_{\theta_{i}, t_{i}}$. Fewer than $r_{0}$ curves can be added to the subcomplex bounded by $\Gamma_{1}$. Thus

$$
\operatorname{card}\left\{\beta \mid \Gamma_{1}\right\}=0\left(\sigma^{-t_{1}}\left(\log \sigma^{-l_{1}}\right)^{k}\right)=0\left(\sigma^{-t_{1}} l_{1}^{k}\right) \text {. }
$$


Thus

$$
\operatorname{card}\{\beta\}=\sum_{l_{1}=M_{1}}^{M_{2} \log m} 0\left(\sigma^{-l_{1}} l_{1}^{k} \sigma^{l} ! m\left(\log \sigma^{l^{\prime}} m\right)^{k}\right)=0\left(m(\log m)^{k}\right)
$$

for a different $k$. This completes the proof under the assumption $\beta$ is $D$-close to $\alpha$.

To complete the proof of Theorem 4.1 we need to consider $\beta$ not $D$-close to $\alpha$. For any such $\beta$ we apply Proposition 1.6 to find a sequence $\beta=\beta_{0}, \ldots, \beta_{n}=\alpha, n \leq$ $C_{1}$, with the property that $\beta_{i}$ is not $M_{1}$-separated from $\beta_{i+1}$ and is $D$-close to it.

The proof is now by induction on $n$. If $n=1, \beta$ is $D$-close to $\alpha$ for which we have the result. Assume the result is true for sequences of length $n \leq n_{0}+1$. Then $\beta_{1}, \ldots, \beta_{n_{0}}=\alpha$ is a sequence of length $n_{0}$. Suppose $\sigma^{l+1} m|\alpha| \leq\left|\beta_{1}\right| \leq \sigma^{\prime} m|\alpha|$.

Since $\left|\beta_{i}\right| \geq\left|\beta_{i+1}\right| / M_{1}$ for each $i$, we have $\left|\beta_{1}\right| \geq|\alpha| / M_{2}$ for some $M_{2}$. Thus $M_{4} \leq l \leq$ $M_{3} \log m$ for universal $M_{3}, M_{4}$. By the induction hypothesis on the length of the sequence,

$$
\operatorname{card}\left\{\beta_{1}\right\}=0\left(\sigma^{\prime} m\left(\log \sigma^{\prime} m\right)^{k}\right)
$$

and since $\beta_{0} D$-close to $\beta_{1}$ and not $M_{1}$-separated from it

$$
\text { card }\left\{\beta_{0} / \beta_{1}\right\}=0\left(\sigma^{-1}\left(\log \sigma^{-1}\right)^{k}\right)
$$

since we have proved the theorem in that case. Thus

$$
\operatorname{card}\left\{\beta_{0}\right\}=\operatorname{card}\left\{\beta_{0} / \beta_{1}\right\} \operatorname{card}\left\{\beta_{1}\right\}=\sum_{l=M_{4}}^{M_{3} \log m} 0\left(\sigma^{-l} l^{k} \sigma^{l} m\left(\log \sigma^{\prime} m\right)^{k}\right)=0\left(m(\log m)^{k}\right. \text {. }
$$

The proof is complete.

Proof of Theorem 1. The number of saddle connections on $X$ of length $\leq 4 C_{2} \varepsilon$ is finite. Thus we may assume $|\beta| \geq 4 C_{2} \varepsilon$. Similarly in what follows we need only consider systems $\geq 4 C_{2} \varepsilon$ in length. Motivated by Proposition 3.1, for any $m$ and just as in the proof of Theorem 4.1 let $V(m)$ be the set of systems $\Gamma$ such that

(a) $\Gamma$ is either a saddle connection or divides $X$ surface into two components,

(b) $4 C_{2} \varepsilon \leq|\Gamma| \leq m$,

(c) for some $(\theta, t) \varepsilon / 2 \leq v_{\theta, t}(\Gamma) \leq|\Gamma|_{\theta, t} \leq \varepsilon$,

(d) for some $t^{\prime} \geq t$, if $\Gamma$ is not a singleton, $|\Gamma|_{\theta, t^{\prime}} \leq C_{2} \varepsilon$ and cannot be $C_{2} \varepsilon$-extended. Claim. card $V(m)=0\left(m^{2}\right)$. To prove the claim, for any $\Gamma_{0} \in V(m)$ consider the sequence $\Gamma_{0}, \Gamma_{1}, \ldots, \Gamma_{\mathrm{p}}$ constructed in Proposition 3.1. The proof is by induction on the length of this sequence.

Let $\gamma_{i}$ be the longest curve on $\Gamma_{i}$. If $p=0, \gamma_{0}$ is already isolated at time $t$. Then card $\left\{\gamma_{0}\right\}$ is $0\left(m^{2}\right)$ by Proposition 2.1 and card $\left\{\Gamma_{0}\right\}$ is $0\left(m^{2}\right)$ by Proposition 2.5.

The induction hypothesis is now: card $\left\{\Gamma_{0} \in V(m)\right\}$ is $0\left(m^{2}\right)$ for the set of $\Gamma_{0}$ that determine sequences $\Gamma_{0}, \ldots, \Gamma_{j}, j \leq p-1$. Now suppose $\Gamma_{0} \in V(m)$ by Proposition 3.1 defines a sequence $\Gamma_{0}, \ldots, \Gamma_{p}$ of length $p+1$. Then $\sigma^{l_{1}+1} m \leq\left|\Gamma_{1}\right| \leq \sigma^{\prime} m$ where $M_{2} \leq l_{1} \leq M_{1} \log m$.

Now either $\left|\Gamma_{1}\right| \leq 4 C_{2} \varepsilon$ or $\left|\Gamma_{1}\right|>4 C_{2} \varepsilon$ in which case $\Gamma_{1} \in V\left(\sigma^{\prime}, m\right)$ and $\Gamma_{1}$ defines a sequence $\Gamma_{1}, \ldots, \Gamma_{p}$ of length $p$. In the first case card $\left\{\Gamma_{1}\right\}$ is $0(1)$. In the second the induction hypothesis applies to give

$$
\text { card }\left\{\Gamma_{1}\right\}=0\left(\sigma^{21} m^{2}\right) \text {. }
$$


By Proposition 3.1 there is a $\gamma_{0} \in \Gamma_{0}$ not $C_{5}$-separated from $\Gamma_{1}$ at time $t=t_{1}$ and satisfying $v_{t_{1}}\left(\gamma_{0}\right) \geq \frac{1}{2} h_{t_{1}}\left(\gamma_{0}\right)$. Suppose

$$
\sigma^{l^{+}+1} m \leq\left|\gamma_{0}\right| \leq \sigma^{\prime} m
$$

As in the proof of Theorem 4.1 we have $0 \leq I_{0} \leq M_{3} l_{1}$. We conclude by Lemma 3.2 that there are $s \leq C_{1}$, pairs $\left(\theta_{i}, t_{i}\right)$ such that for any $\gamma_{0}$ there is a pair $\left(\theta_{i}, t_{i}\right)$ such that

$$
\frac{\left|\gamma_{0}\right|_{\theta_{i}, t_{i}}}{\left|\Gamma_{1}\right|_{\theta_{i}, t_{i}}} \text { is } 0\left(\frac{\left|\gamma_{0}\right|}{\left|\Gamma_{1}\right|}\right)=0\left(\sigma^{l^{t}-l_{1}}\right) \text {. }
$$

On the surface $Y_{\theta_{i}, r_{i}}$ renormalize the complex with boundary $\Gamma_{1}$ so it has area 1 . Then by Theorem 4.1 ,

$$
\operatorname{card}\left\{\gamma_{0} \mid \Gamma_{1}\right\}=0\left(\sigma^{l^{-}-l_{1}}\left(l_{1}-l_{0}\right)^{k}\right)
$$

and so

$$
\operatorname{card}\left\{\Gamma_{0} \mid \Gamma_{1}\right\}=0\left(\sigma^{l_{0}-l_{1}}\left(l_{1}-l_{0}\right)^{k} \sigma^{-l_{0}}\right)=0\left(\sigma^{-l_{1}}\left(l_{1}-l_{0}\right)^{k}\right)
$$

by Proposition 2.5. Thus

$$
\text { card } \begin{aligned}
V(m) & =\sum_{l_{1}=M_{2}}^{M_{1} \log m} \sum_{l_{0}=0}^{M_{3} l_{1}} \operatorname{card}\left\{\Gamma_{0} \mid \Gamma_{1}\right\} \operatorname{card}\left\{\Gamma_{1}\right\} \\
& =\sum_{l_{1}=M_{2}}^{M_{1} \log m} \sum_{l_{0}=0}^{M_{3} l_{1}} 0\left(\sigma^{l_{1}} m^{2}\left(l_{1}-l_{0}\right)^{k}\right) \\
& \leq 0\left(m^{2} \sum_{l_{1}=M_{2}}^{M_{1} \log m} \sigma^{l^{\prime}} l_{1}^{k}\right)=0\left(m^{2}\right)
\end{aligned}
$$

since $\sum_{l=M_{2}}^{\infty} \sigma^{\prime} l^{k}$ converges for fixed $k, M_{2}$. This proves the claim.

Finally we compute card $S_{\sigma}(n)$. Card $S_{\sigma}^{\varepsilon}(n)$ is $0\left(n^{2}\right)$ so we may assume $\beta \in S_{\sigma}(n)$ is not $\varepsilon$-wide. We may assume $|\beta| \geq 4 C_{2} \varepsilon$. For each $\beta$ we form the sequence $\beta=\Gamma_{0}, \Gamma_{1}, \ldots, \Gamma_{p}$ given by Proposition 3.1. Then $\Gamma_{1} \in V\left(\sigma^{\prime}, n\right)$ where $M_{2} \leq l_{1} \leq$ $M_{1} \log n$. By the claim, card $V\left(\sigma^{l} n\right)$ is $0\left(\sigma^{2 l} n^{2}\right)$. Then by Lemma 3.2 and Theorem 4.1 just as in the proof of the claim,

$$
\text { card }\left\{\beta \mid \Gamma_{1}\right\} \text { is } 0\left(\sigma^{-l_{1}}\right) l_{1}^{k} \text {. }
$$

Then card $\{\beta\}$ is $\sum_{l_{1}=M_{2}}^{M_{1} \log n} \sigma^{-l_{1}} l_{1}^{k} 0\left(\sigma^{2 l_{1}} n^{2}\right)$ which is $0\left(n^{2}\right)$ since $\sum_{l_{1}=1}^{\infty} \sigma^{l_{1}} l_{1}^{k}<\infty$.

\section{REFERENCES}

[B] M. Boshennitzan. A condition for minimal interval exchange maps to be uniquely ergodic. Duke Math. J. 52 (1985), 723-752.

[G] E. Gutkin. Billiards on almost integrable polyhedral surfaces. Ergodic Th. \& Dynam. Sys. 4 (1984), 569-584.

[K] A. Katok. The growth rate for the number of singular and periodic orbits for a polygonal billiard. Preprint.

[K-M-S] S. Kerckhoff, H. Masur \& J. Smillie. Ergodicity of billiard flows and quadratic differentials. Ann. Math. 124 (1986), 293-311.

[R] M. Rees. An alternative approach to the ergodic theory of measured foliations on surfaces. Ergod. Th. \& Dynam. Sys. 1 (1981), 461-488.

[St] K. Strebel. Quadratic Differentials. Springer-Verlag: New York, 1984.

[Su] D. Sullivan. Disjoint spheres, approximation by imaginary quadratic numbers and the logarithm law for geodesics. Acta Math. 149 (1982), 215-238. 